\title{
Stochastic Hydrodynamics
}

\author{
Weinan E
}

\begin{abstract}
We review the progress made recently on the mathematical understanding of prototypical stochastic partial differential equations in fluid mechanics, including the stochastic Burgers equation, stochastic passive scalar equations and stochastic Navier-Stokes equation, with particular emphasis on issues related to the problem of turbulence. Questions addressed include the existence and uniqueness of invariant measures, construction of the invariant measures and typical behavior of stationary solutions. Also discussed is the interplay between regularity and dissipation in the inviscid limit. The concepts and methods reviewed here include the one force - one solution principle, variational methods, master equations, generalized flows, reduction to Gibbsian dynamics, hypoellipticity, etc.
\end{abstract}

\section{Contents}

1. Introduction

2. Stochastic Burgers Equation 112

2.1. Invariant Measures 113

2.2. Statistical Theory 116

2.3. Stochastic Hamilton-Jacobi Equation in High Dimensions 121

3. Stochastic Passive Scalar Equation 121

3.1. A Simple Example of Generalized Flows 124

3.2. Delta-correlated Gaussian Velocity Fields 124

$\begin{array}{ll}\text { 3.3. Invariant Measures } & 128\end{array}$

3.4. Correlation Functions and Zero Modes 131

4. Stochastic Navier-Stokes Equation 133

4.1. Ergodicity When All Determining Modes are Forced 133

4.2. General Stochastic Dissipative PDEs 137

4:3. Ergodicity with Minimum Stochastic Forcing 137

5. Regularity and Dissipation 141

6. Conclusions 143

Based on talks given at the meeting: Current Development in Mathematics, Harvard University, December 2000 . 


\section{Introduction}

The fundamental problem in stochastic hydrodynamics is the problem of turbulence, which is widely regarded as the last unsolved problem in classical physics. The physical issues are very well summarized in the book of Monin and Yaglom "Statistical Fluid Mechanics" [86]. The mathematical issues, together with a report on recent progress on the understanding of these issues, are the subject of this review.

We start with a physical description of the problem. We are interested in the dynamics of a viscous fluid governed by the Navier-Stokes equation

$$
\left\{\begin{array}{l}
\mathbf{u}_{t}+(\mathbf{u} \cdot \nabla) \mathbf{u}+\nabla p=\nu \Delta \mathbf{u}+\mathbf{f} \\
\nabla \cdot \mathbf{u}=0
\end{array}\right.
$$

Here $\mathbf{u}=\left(u_{1}, u_{2}, u_{3}\right)$ is the velocity field, $p$ is the pressure, $\mathbf{f}$ represents the forcing on the fluid, $\nu$ is the viscosity of the fluid. It is helpful to non-dimensionalize the system (1). Let $U, L$ be the typical velocity and length scale respectively. Define $T=\frac{L}{U}$, and perform a change of variable $\mathbf{x}=\mathbf{x}^{\prime} L, t=t^{\prime} T, \mathbf{u}=\mathbf{u}^{\prime} U$. Omitting the primes, we get

$$
\left\{\begin{array}{l}
\mathbf{u}_{t}+(\mathbf{u} \cdot \nabla) \mathbf{u}+\nabla p=\frac{1}{R e} \Delta \mathbf{u}+\mathbf{f} \\
\nabla \cdot \mathbf{u}=0
\end{array}\right.
$$

where $R e=\frac{U L}{\nu}$ is the dimensionless Reynolds number, which is the key parameter in our problem. We are interested in the situation when $R e$ is very large. Take a typical car on the highway, we have $U=65$ miles $/$ hour $\doteq 3000 \mathrm{~cm} / \mathrm{s}, L=$ length of the car $\doteq 800 \mathrm{~cm}, \nu=$ viscosity of air $=0.132 \mathrm{~cm}^{2} / \mathrm{s}$, which gives $R e \doteq 1.8 \times 10^{7}$.

In a high Reynolds number flow, the most ubiquitous phenomenon is the presence of a wide range of length scales and a hierarchy of eddies (swirls, vortices). The size of the range of scales was estimated by Kolmogorov $[\mathbf{5 9}, \mathbf{6 0}]$ to be $O\left(R e^{3 / 4}\right)$, on the assumption that there is a disparity between the length scales at which energy is supplied to the system, and the scale $\ell_{d}$ at which energy is dissipated through viscosity - the viscous cut-off scale. In between these two scales, energy is transported but not dissipated, resulting in a constant energy flux $\bar{\varepsilon}$. This range is called the inertial range.

Kolmogorov further postulated that the inertial range properties of a fully developed turbulent flow is universal. Using a scaling argument, he predicted that

$$
\left\langle|\mathbf{u}(\mathbf{x}+\mathbf{r}, t)-\mathbf{u}(\mathbf{x}, t)|^{n}\right\rangle \sim C_{n}|\mathbf{r}|^{\frac{n}{3}} \bar{\varepsilon}^{\frac{n}{3}}
$$

for $\ell_{d} \ll|\mathbf{r}| \ll L$, where $C_{n}$ should be universal constants, $\langle\quad\rangle$ means either time average or ensemble average over many realizations of the experiment. (3) means that typical turbulent velocity fields are Hölder continuous with exponent $1 / 3$. 
Most notable special cases of (3) are found when $n=2$ and 3 . When $n=2$, one can represent (3) in Fourier space. Let $\hat{\mathbf{u}}$ be the Fourier transform of $\mathbf{u}$, and $E(k)=\left\langle\sum_{k<|\mathbf{k}| \leq k+1}|\hat{\mathbf{u}}(\mathbf{k})|^{2}\right\rangle$ be the energy spectrum. Then (3) translates to

$$
E(k) \doteq C_{k} \bar{\varepsilon}^{\frac{2}{3}} k^{-\frac{5}{3}}, L^{-1} \ll k \ll k_{d}=\ell_{d}^{-1}
$$

This is the well-known $-\frac{5}{3}$ spectrum. When $n=3$, Kolmogorov derived, from the Navier-Stokes equation, the relation

$$
\left\langle\left(u_{\|}(\mathbf{x}+\mathbf{r}, t)-u_{\|}(\mathbf{x}, t)\right)^{3}\right\rangle \sim-\frac{4}{5} \bar{\varepsilon}|\mathbf{r}|
$$

for $\ell_{d} \ll|\mathbf{r}| \ll L$, assuming that the inertial range properties are homogenous and isotropic. Here $u_{\|}$is the component of the velocity field parallel to $r$. It is worth emphasizing that (5) is among the very few results in turbulence theory that are actually derived from the Navier-Stokes equation.

Kolmogorov's theory was immediately challenged by Landau [48], who argued that dissipation processes in turbulence are tied with the non-universal large scales, hence the small scale inertial range properties cannot be truly universal. In reality, dissipation is intermittent, dominated by strong but rare events. This means that there are nontrivial corrections, to a mean-field theory such as Kolmogorov's due to fluctuation. Specifically, (3) should be changed to

$$
\left\langle|\mathbf{u}(\mathbf{x}+\mathbf{r}, t)-\mathbf{u}(\mathbf{x}, t)|^{n}\right\rangle \sim C_{n} L^{\frac{n}{3}-\alpha_{n}}|\mathbf{r}|^{\alpha_{n}} \bar{\varepsilon}^{\frac{n}{3}}
$$

$\mathbf{u}$ is said to have multi-fractal scaling if $\alpha_{n}$ is not linear in $n$. Work on such intermittency corrections has been a central theme in modern turbulence theory $[3,4,20,48,61]$.

We now turn to the mathematical formulation of the problem. To begin with, we have to understand the sense of averaging, the bracket \langle\rangle . Since a turbulent flow is intrinsically unstable and stochastic - a small perturbation in the initial data leads to large differences in the solution, it is natural to use a statistical description. Therefore we will be interested in statistical steady states, the invariant measures of the Navier-Stokes equation (2). We can now formulate the mathematical questions as follows.

1. The existence and uniqueness of an invariant measure for the dynamics of (2), for both finite and infinite Reynolds numbers. The existence of an invariant measure at infinite Reynolds number ensures that there is sufficient dissipation in the system even in the limit of zero viscosity. Uniqueness guarantees that the dynamics is ergodic in the phase space, as is commonly assumed in turbulence theory.

2. Let $\mu_{0}$ be the invariant measure of (2) at infinite Reynolds number. We can then study the regularities of the statistically stationary solutions. In particular, we can study the decay of their energy spectrum.

At the present time, neither question is answered. Worse than that, we still face the well-known problem that uniqueness of solutions to the three-dimensional Navier-Stokes equation is still yet to be proved, leaving us with no starting point to address these questions.

However, it is important to realize that, from a physical viewpoint, the problem of hydrodynamic turbulence is just one of many problems exhibiting turbulent behavior, e.g. a wide range of active scales, slow algebraic decay of the energy 
spectrum, intermittent dissipation processes, etc. The mathematical issues discussed above are equally important in these problems. A list of such "generalized turbulence problems" include:

1. Two-dimensional hydrodynamic turbulence.

2. Wave turbulence.

3. Shell models of turbulence.

4. Burgers turbulence.

5. Turbulent transport of passive scalars.

These problems are closely related to the original turbulence problem and are expected to be simpler to deal with. There are many more such problems in nonequilibrium statistical physics outside the traditional areas of hydrodynamics. However, here we will restrict ourselves to problems in hydrodynamics.

Below we will discuss progress made recently on the problems 1, 4 and 5. Our basic strategy is to consider the appropriate PDEs under a large scale stochastic forcing and study the properties of the statistical steady states. For problems 4 and 5 , we obtain rather precise descriptions of the small scales. For problem 1, we prove ergodicity of the dynamics.

Before proceeding further, let us remark on the stochastic setting of our approach. Traditionally problems in PDEs have been studied using deterministic methods. However, deterministic methods usually predict the worst-case scenario and it is awkward to avoid atypical behavior such as starting from an unstable fixed point. Therefore if our main interest is the typical behavior of solutions of a complex system in phase space, it is much more advantageous to use a stochastic setting which provides a natural framework to describe typical behavior of the solutions. On the other hand, it is important, in a stochastic setting, to distinguish results that are tied with the randomness in the formulation and those that are intrinsic properties of the underlying PDEs.

\section{Stochastic Burgers Equation}

In this section, we consider the Burgers equation with stochastic forcing

$$
\frac{\partial u}{\partial t}+u \frac{\partial u}{\partial x}=\nu \frac{\partial^{2} u}{\partial x^{2}}+f
$$

Besides being a prototype for nonlinear waves, (7) is also a canonical example in non-equilibrium statistical physics. As such, it describes the statistical mechanics of strings in a random potential. The string is assumed to be directed, i.e. there exists a time axis such that the position of the string is a single-valued function over this time axis. Vortex lines in high temperature superconductors [10], charge density waves [43], directed polymers and stochastic interfaces in $1+1$ dimensional SOS models [58] are all examples of such strings. To see this connection, define the partition function for the configurations of the strings over the time interval $[0, t]$ assuming that they are pinned at time $t$ at location $x$ :

$$
Z(x, t)=\left\langle\exp \left(-\beta \int_{0}^{t}\left(\frac{1}{2}|\dot{\xi}(\tau)|^{2}+V(\xi(\tau), \tau)\right) d \tau\right) \mid \xi(t)=x\right\rangle,
$$

where $\beta=1 / k T, k$ is the Boltzmann constant, $T$ is the temperature. The averaging \langle\rangle is over all path $\xi$ such that $\xi(t)=x$. The first term in the exponent is the elastic energy and the second term is the potential energy with $V$ being the potential. The 
associated free energy $\varphi=k T \log Z$ satisfies

$$
\frac{\partial \varphi}{\partial t}+\frac{1}{2}|\nabla \varphi|^{2}=k T \Delta \varphi+V
$$

In one dimension, let $u=\partial \varphi / \partial x$, we obtain (8) with $\nu=k T$. When $V$ is the space-time white noise, (9) is the well-known KPZ equation [58].

2.1. Invariant Measures. The existence of an invariant measure for (2) is only understood so far on finite domains. Consider, for example, $(7)$ on $[0,2 \pi]$ with periodic boundary condition. We will sometimes identify the domain as $S^{1}$, the unit circle. The forcing function can be expressed as:

$$
f(x, t) d t=\sum_{k} f_{k}(x) d W_{k}(t)
$$

where the $\left\{W_{k}(\cdot)\right\}$ 's are independent Wiener processes. We will assume that

$$
f_{k}(\cdot) \in C^{3}\left(S^{1}\right), \quad\left|\frac{\partial f_{k}}{\partial x}(x)\right| \leq \frac{C}{k^{2}}
$$

for all $k$. We will use $(\Omega, \mathcal{F}, P)$ to denote the probability space for the forcing functions $f$, and $\omega \in \Omega$ to denote a typical realization of the force. $\mathcal{F}_{t}$ denotes the $\sigma$-algebra generated by the forces up to time $t$.

When $\nu=0,(7)$ is understood in the weak sense with solutions satisfying the entropy condition. The precise definition for the random case is given in [30]. In this case, we write (7) as

$$
\frac{\partial u}{\partial t}+\frac{1}{2} \frac{\partial}{\partial x} u^{2}=f
$$

In accordance with the conservative nature of the Burgers equation, we will assume that the $f_{k}$ 's satisfy: $\int_{S^{1}} f_{k}(x) d x=0$. Then (12) admits a conservation law: $\int_{S^{1}} u(x, t) d x=\int_{S^{1}} u(x, 0) d x$. Without loss of generality, we will assume $\int_{S^{1}} u(x, t) d x=0$ and restrict ourselves to the space of functions satisfying this condition.

A natural phase space for (12) is the Skorohod space $D$ on $S^{1}$ which consists of functions admitting only discontinuities of the first kind [9]. Let $\mathcal{D}$ be the Borel $\sigma$-algebra on $D$. (12) can then be viewed as a Markov process on $D$ with transition probability

$$
P_{t}(u, A)=\int_{\Omega} \chi_{A}(u, \omega) P(d \omega)
$$

where $u \in D, A \in \mathcal{D}$, and

$$
\chi_{A}(u, \omega)= \begin{cases}1 & \text { if } S_{\omega}(t) u \in A \\ 0 & \text { otherwise }\end{cases}
$$

Here $S_{\omega}(t)$ denotes the solution operator of (12) at time $t$ with forcing $\omega$.

Definition 1. An invariant measure $\mu_{0}(d u)$ of the Markov process (12) is a measure on $(D, \mathcal{D})$ satisfying

$$
\mu_{0}(A)=\int_{D} P_{t}(u, A) \mu_{0}(d u)
$$

for any $A \in \mathcal{D}$ and any $t>0$.

THEOREM 2. [30]. (12) admits a unique invariant measure on the space $D$. 
The proof of Theorem 2 was based on the following variational characterization of weak solutions of (12) satisfying the entropy condition. Let $\xi:\left[t_{1}, t_{2}\right] \rightarrow R^{1}$ be a Lipschitz continuous curve. Define the action functional

$$
\begin{aligned}
\mathcal{A}_{t_{1}, t_{2}}(\xi) & =\int_{t_{1}}^{t_{2}}\left(\frac{1}{2}(\dot{\xi}(s))^{2}-\sum_{k} f_{k}(\xi(s)) \dot{\xi}(s)\left(W_{k}(s)-W_{k}\left(t_{1}\right)\right)\right) d s \\
& +\sum_{k} f_{k}\left(\xi\left(t_{2}\right)\right)\left(W_{k}\left(t_{2}\right)-W_{k}\left(t_{1}\right)\right)
\end{aligned}
$$

Then we have for $\tau<t$,

$$
u(x, t)=\frac{\partial}{\partial x} \inf _{\xi(t)=x}\left\{\mathcal{A}_{\tau, t}(\xi)+\int_{0}^{\xi(\tau)} u(z, \tau) d z\right\}
$$

Minimizers of the functional in (16) satisfy the following Euler-Lagrange equation:

$$
d \gamma(s)=v(s) d s, \quad d v(s)=\sum_{k=1}^{\infty} f_{k}(\gamma(s)) d W_{k}(s)
$$

Using (17), we can construct solutions of (12) as follows. Fix $t$. For values of $x$ such that the minimizer to the functional (16) is unique, say $\xi(\cdot)$, then $u(\cdot, t)$ is continuous at $x$, and $u(x, t)=\dot{\xi}(t)$. On the other hand, for values of $x$ such that the minimizers to the variational problem are not unique, then $u(\cdot, t)$ is discontinuous at $x$ with $u(x+, t)=\inf _{\alpha}\left\{\dot{\xi}_{\alpha}(t)\right\}$ and $u(x-, t)=\sup _{\alpha}\left\{\dot{\xi}_{\alpha}(t)\right\}$ where $\left\{\xi_{\alpha}\right\}$ denotes the family of minimizers of (16) such that $\xi_{\alpha}(t)=x$.

This construction is just a reformulation of the method of characteristics for weak solutions. It is a variational formulation of the backward characteristics defined in [23]. In particular, the Euler-Lagrange equation (18) is nothing but the equation for the characteristics of (12).

Of particular interest to the construction of the invariant measure is a special class of minimizers called the one-sided minimizers (OSM).

Definition 3. A piecewise $C^{1}$-curve $\xi:(-\infty, 0] \rightarrow S^{1}$ is a one-sided minimizer if for any Lipschitz continuous $\tilde{\xi}:(-\infty, 0] \rightarrow S^{1}$ such that $\tilde{\xi}(0)=\xi(0)$ and $\tilde{\xi}=\xi$ on $(-\infty, \tau]$ for some $\tau<0$, we have

$$
\mathcal{A}_{s, 0}(\xi) \leq \mathcal{A}_{s, 0}(\tilde{\xi})
$$

for all $s \leq \tau$. Similarly, we define one-sided minimizers on $(-\infty, t]$, for $t \in R^{1}$.

Next we ask the following question: Given $(x, t)$, how many OSMs $\xi$ exist such that $\xi(t)=x$ ? This question is answered by studying the intersection properties of OSMs. As a general fact in the calculus of variations, two different OSMs cannot intersect more than once. In other words, if $\xi_{1}, \xi_{2}$ are two OSMs that there exist $t_{1}, t_{2} \in R^{1}, t_{1} \neq t_{2}$, and $\xi_{1}\left(t_{1}\right)=\xi_{2}\left(t_{1}\right), \xi_{1}\left(t_{2}\right)=\xi_{2}\left(t_{2}\right)$, then $\xi_{1} \equiv \xi_{2}$ on their common domain of definition [80]. However, more is true in the random case. If $\xi_{1}$ and $\xi_{2}$ are two different OSMs, such that $\xi_{1}(s)=\xi_{2}(s)$ for some $s$, then neither $\xi_{1}$ nor $\xi_{2}$ can be extended as an OSM beyond the interval $(-\infty, s]$. This is because that in the random case, two OSMs always have an effective intersection at $t=-\infty$. The precise formulation of this property is given in [30].

These intersection properties have far-reaching consequences. Let us fix $t=0$. By considering the image of all OSMs at $t=-1$, it is easy to see that the set

$$
I_{\omega}=\left\{x \in S^{1}: \text { there exist more than one OSM } \xi \text { such that } \xi(0)=x\right\}
$$


can at most be a countable set for almost all $\omega \in \Omega$. Therefore we can define:

$$
u_{\omega}(x, 0)=\dot{\xi}(0),
$$

where $\xi$ is the OSM such that $\xi(0)=x . u_{\omega}(\cdot, 0)$ is a well-defined function in $L^{\infty}\left(S^{1}\right)$ for almost all $\omega$.

Similar construction can be carried out for any $t \in R^{1}$ which defines $u_{\omega}(\cdot, t)$. Furthermore it is easy to conclude from the variational principle (17) that

$$
u_{\omega}(\cdot, t)=S_{\omega}(t) u_{\omega}(\cdot, 0),
$$

for $t>0$. In other words, $u_{\omega}$ is a solution of (12). This is a special solution of (12) for the given realization of the force $\omega$. This construction is an example of the so-called "one force, one solution" principle, namely for almost every realization of the force, we can associate one and only one special solution to that force. In other words, the random attractor consists of one and only one trajectory. It is straightforward to check that the distribution of the mapping:

$$
\Phi_{0}: \omega \rightarrow u_{\omega}(\cdot, 0) \text {, }
$$

is an invariant measure for (12). Moreover $\Phi$ satisfies the invariance principle:

$$
\Phi_{0}\left(\theta_{t} \omega\right)=S_{\omega}(t) \Phi_{0}(\omega)
$$

Therefore we have

THEOREM 4. [30]. There exists an invariant mapping $\left\{\Phi_{0}\right\}: \Omega \rightarrow D$ satisfying (23). Furthermore the invariant measure $\mu_{0}$ is the distribution of $\Phi_{0}$.

Theorem 4 states that the statistically stationary solutions are functionals of the forcing. Uniqueness of the invariant measure follows from the fact that the OSMs are largely unique.

It is shown in $[\mathbf{9 3}, 94]$ that for $\nu>0,(12)$ also admits a unique invariant mapping $\Phi_{0}^{\nu}$ whose distribution is the unique invariant measure for (12), denoted by $\mu_{\nu}$. We have

THEOREM 5. [30].

$$
\Phi_{0}^{\nu}(\omega) \rightarrow \Phi_{0}(\omega)
$$

in $L^{1}\left(S^{1}\right)$ for almost every $\omega \in \Omega$. Consequently

$$
\mu_{\nu} \rightarrow \mu_{0}
$$

weakly.

We remark that (24), (25) are a different kind of convergence statement from standard theorems on zero-viscosity limits of (12) studied in the PDE literature $[95,97]$. There we are given a sequence of initial data that converge in the inviscid limit, and we ask whether convergence still holds at later times. Here we are not given any initial data, and we proceed to establish convergence with the only information that the solutions are defined for all $t \in R^{1}$ in a special way using the OSMs. Consequently the techniques used to prove Theorem 5 are very different from the ones used in the PDE literature to study inviscid limits. See [30].

Our next task is to characterize the statistically stationary solutions. This requires a non-degeneracy condition to the effect that the process (18) starting at any $x \in S^{1}$ is transitive on $S^{1}$. This condition is generically satisfied. However it is violated if the sum in (10) contains only one term. We refer to [30] for a 
detailed formulation and examination of this condition. Under this non-degeneracy condition, we have

THEOREM 6. [30]. For almost all $\omega, u_{\omega}$ satisfies the following:

1. There exists a unique two-sided minimizer (TSM, defined below) $y_{\omega}: R^{1} \rightarrow$ $S^{1}$ which is a characteristic of (12) associated with the solution $u_{\omega}$.

2. There exists a unique so-called main shock $\gamma_{\omega}: R^{1} \rightarrow S^{1}$, which is a continuous shock curve defined for all $t \in R^{1}$.

3. The TSM is a hyperbolic trajectory for the dynamical systems (18).

4. For any $t \in R^{1}$, there exist global stable and unstable manifolds associated with $y_{\omega}$ at time $t$, denoted by $W_{\omega}^{s}(t)$ and $W_{\omega}^{u}(t)$ respectively, on the phase space $S^{1} \times R^{1}$.

5. The graph of $u_{\omega}(\cdot, t)$ is a subset of $W_{\omega}^{u}(t)$.

As a corollary, we have that for each fixed $t, u_{\omega}$ is almost surely a piecewise smooth function.

Definition 7. A piecewise $C^{1}$-curve $\xi:(-\infty,+\infty) \rightarrow S^{1}$ is a two-sided minimizer if for any Lipschitz continuous $\tilde{\xi}:(-\infty,+\infty) \rightarrow S^{1}$ such that $\tilde{\xi}=\xi$ away from a compact set, we have

$$
\mathcal{A}_{-s, s}(\xi) \leq \mathcal{A}_{-s, s}(\tilde{\xi})
$$

for large enough s.

The possibility of establishing hyperbolicity of TSMs in the random case comes from the following:

Basic Collision Lemma 8. Assuming that the non-degeneracy condition holds. Then there exists a constant $p_{0}$, depending only on the $\left\{f_{k}\right\}$ 's, with the following property: Given an arbitrary pair of points $\left(x_{1}, x_{2}\right) \in[0,1]^{2}$ at $t=0$ whose positions are $\mathcal{F}_{0}$-measurable,

$$
P\left\{x_{1}, x_{2} \text { merge before } t=1\right\}>p_{0} .
$$

Heuristically two points merge before $t=1$ if the forward characteristics emanating from them intersect before $t=1$. This of course depends on the forces as well as the solution at $t=0$. The lemma states that independent of the solution at $t=0$, one can always find a set of forces with positive measure under which the two points merge.

The proof of the Basic Collision Lemma relies on PDE techniques and is given in the Appendix D of [30].

The Basic Collision Lemma provides the mechanism for the origin of the hyperbolicity. In particular, the uniqueness of the TSM and the main shock is a simple consequence of the Basic Collision Lemma.

The regularity and structural properties described here are used in $[29,34,35]$ to study the statistical behavior of the Burgers equation. A summary of these results is given below.

2.2. Statistical Theory. We now address the questions frequently asked in the physics literature regarding (12), building upon the regularity results described in $\S 2.1$. Since we have established the existence of $\mu_{0}$ which is the statistical steady state at the inviscid limit, we can restrict our attention to this case. We warn the 
reader that some of the results described in this section are not fully rigorously proved. This section is a summary of $[34,35]$

We will denote by $B$ the spatial correlation function of the forcing:

$$
\langle f(x, t) f(y, s)\rangle=2 B(x-y) \delta(t-s)
$$

2.2.1. Structure Functions. The fact that $u_{\omega}$ is piecewise smooth implies easily that

$$
S_{p}(r)=\left\langle|u(x+r)-u(x)|^{p}\right\rangle=\left\{\begin{array}{l}
r^{p}\left\langle|\xi|^{p}\right\rangle+o\left(r^{p}\right) \text { if } 0 \leq p<1 \\
r \rho\left\langle|s|^{p}\right\rangle+o(r) \text { if } 1<p
\end{array}\right.
$$

where $\rho$ is the number density of the shocks, which is finite from the results described earlier, $s$ is the jump across the shocks, $\xi$ is the regular part of the velocity gradient:

$$
\frac{\partial}{\partial x} u(x, t)=\xi(x, t)+\sum_{j} s\left(y_{j}, t\right) \delta\left(x-y_{j}\right),
$$

with $\xi(\cdot, t) \in L^{1}\left(S^{1}\right)$.

If we write $S_{p}(r)=C_{p} r^{\alpha_{p}}$, for $r \ll 1$, then $\alpha_{p}=p$ for $0 \leq p<1$, and $\alpha_{p}=1$, for $p \geq 1$. This is a reflection of the fact that as far as regularity is concerned, almost everywhere the solution is either Lipschitz continuous or discontinuous. The linear part in the graph of $\alpha_{p}$ probes the Lipschitz continuous part of the solution. The flat part probes the discontinuous part of the solution. Such a situation is sometimes referred to as "bifractal".

2.2.2. Velocity Gradient PDF. More difficult are the questions of probability density functions (PDFs) for quantities such as $u, \partial u / \partial x$ and $\delta u(x, t)=u(x+$ $y, t)-u(y, t)$. In particular, the PDF of $\xi=\partial u / \partial x$ (suitably defined), $Q(\xi)$, has attracted a lot of attention in recent years. In the inviscid limit, it is agreed that $Q(\xi)$ has the behavior

$$
Q(\xi) \sim \begin{cases}C_{-}|\xi|^{-\alpha} & \text { as } \xi \rightarrow-\infty, \\ C_{+} \xi^{\beta} \mathrm{e}^{-\xi^{3} /\left(3 B_{1}\right)} & \text { as } \xi \rightarrow+\infty .\end{cases}
$$

where $C_{-}, C_{+}$are constants, $B_{1}=-B_{x x}(0)$. But many different values of $\alpha$ and $\beta$ have been proposed (see [35] for a review).

A priori, there is even an issue how to define $Q$. In the inviscid limit one can define the PDF for the velocity divided difference $(u(y+\delta, t)-u(y, t)) / \delta, Q^{\delta}(\xi, t)$ and then take the limit as $\delta \rightarrow 0$. An alternative is to first define the PDF of $\partial u / \partial x$ for the viscous problem, call it $Q^{\nu}(\xi, t)$, and then take the limit as $\nu \rightarrow 0$ :

$$
\underline{Q}(\xi, t)=\lim _{\nu \rightarrow 0} Q^{\nu}(\xi, t), \quad Q(\xi, t)=\lim _{\delta \rightarrow 0} Q^{\delta}(\xi, t) .
$$

While a fully rigorous proof was not constructed, [35] presented strong evidence that for the case studied here,

$$
Q=\underline{Q}
$$

Below we will concentrate on $Q$.

Using the calculus for functions of bounded variation [101], we can derive an equation for $Q$

$$
\frac{\partial Q}{\partial t}=\xi Q+\frac{\partial}{\partial \xi}\left(\xi^{2} Q\right)+B_{1} \frac{\partial^{2} Q}{\partial \xi^{2}}+F(\xi, t)
$$


where

$$
F(\xi, t)=\rho \int_{-\infty}^{0} s V(s, \xi, t) d s \leq 0 .
$$

Here $V(s, \xi, t)=V_{+}(s, \xi, t)+V_{-}(s, \xi, t), V_{ \pm}\left(s, \xi_{ \pm}, t\right)$ are the PDFs of $\left(s(y, t), \xi_{ \pm}(y, t)=\right.$ $\partial u(y \pm, t) / \partial y)$ conditional on $y$ being a shock position. This equation should be compared with the equation for $Q^{\nu}$ :

$$
\frac{\partial Q^{\nu}}{\partial t}=\xi Q^{\nu}+\frac{\partial}{\partial \xi}\left(\xi^{2} Q^{\nu}\right)+B_{1} \frac{\partial^{2} Q^{\nu}}{\partial \xi^{2}}-\nu \frac{\partial}{\partial \xi}\left(\left\langle\frac{\partial^{2} \xi}{\partial x^{2}} \mid \xi\right\rangle Q^{\nu}\right)
$$

where $\left\langle\partial^{2} \xi / \partial x^{2} \mid \xi\right\rangle$ is the ensemble-average of $\partial^{2} \xi / \partial x^{2}$ conditional on $\xi$. We see that

$$
F(\xi, t)=-\lim _{\nu \rightarrow 0} \nu \frac{\partial}{\partial \xi}\left(\left\langle\frac{\partial^{2} \xi}{\partial x^{2}} \mid \xi\right\rangle Q^{\nu}\right)
$$

Even though we are primarily interested in statistical steady states, we have written down the master equations for the more general case including transients.

Integrating (33), we get

$$
\frac{d}{d t} \int_{R^{1}} Q(\xi, t) d \xi=\langle\xi\rangle+\rho\langle s\rangle=0
$$

Consequently

$$
\lim _{\nu \rightarrow 0} \int_{R^{1}} \xi Q^{\nu}(\xi, t) d \xi \neq \int_{R^{1}} \xi Q(\xi, t) d \xi,
$$

since the left hand side vanishes due to homogeneity.

Even though (33) is not a closed equation since the form of $F$ is unknown, we can already obtain from it non-trivial information. As an example, we have

$$
\lim _{|\xi| \rightarrow+\infty}|\xi|^{3} Q(\xi, t)=0
$$

i.e. $Q$ decays faster than $|\xi|^{-3}$ as $\xi \rightarrow-\infty$.

This result rules out all the proposed value of $\alpha$ except that of [29] which gives $\alpha=7 / 2$.

(38) is obtained by combining (36) together with the following:

1. Take the first moment of (33) gives

$$
\frac{d}{d t}\langle\xi\rangle=\left[\xi^{3} Q\right]_{-\infty}^{+\infty}+\frac{\rho}{2}\left(\left\langle s \xi_{-}\right\rangle+\left\langle s \xi_{+}\right\rangle\right)
$$

2. Along the shock, we have

$$
\frac{d}{d t}(\rho\langle s\rangle)=-\frac{\rho}{2}\left(\left\langle s \xi_{-}\right\rangle+\left\langle s \xi_{+}\right\rangle\right)
$$

which is a consequence of the equations for the dynamics of the shocks.

3. By the definition of $F$,

$$
\int_{R^{1}} \xi F(\xi, t) d \xi=\frac{\rho}{2}\left(\left\langle s \xi_{-}\right\rangle+\left\langle s \xi_{+}\right\rangle\right)
$$

It can also be shown that $\xi F(\xi, t)$ is absolutely integrable on $R^{1}$ for all $t$. 
2.2.3. Asymptotics for the Statistical Stationary State. In statistical steady state, (33) becomes

$$
\xi Q+\frac{d}{d \xi}\left(\xi^{2} Q\right)+B_{1} \frac{d^{2} Q}{d \xi^{2}}+F(\xi)=0 .
$$

This is a second order ODE with an inhomogeneous term $F$. We can write the general solutions of (39) as

$$
Q=Q_{s}+C_{1} Q_{1}+C_{2} Q_{2}
$$

where $Q_{1}$ and $Q_{2}$ are two linearly independent solutions of the homogeneous equation, and $Q_{s}$ is a particular solution. For example, we can take:

$$
\begin{gathered}
Q_{1}(\xi)=\xi \mathrm{e}^{-\Lambda} \\
Q_{2}(\xi)=1-\frac{\xi \mathrm{e}^{-\Lambda}}{B_{1}} \int_{-\infty}^{\xi} \xi^{\prime} \mathrm{e}^{\Lambda^{\prime}} d \xi^{\prime} \\
Q_{s}(\xi)=\frac{1}{B_{1}} \int_{-\infty}^{\xi} \xi^{\prime} F\left(\xi^{\prime}\right) d \xi^{\prime}-\frac{\xi \mathrm{e}^{-\Lambda}}{B_{1}} \int_{-\infty}^{\xi} \mathrm{e}^{\Lambda^{\prime}} G\left(\xi^{\prime}\right) d \xi^{\prime},
\end{gathered}
$$

where

$$
\Lambda=\frac{\xi^{3}}{3 B_{1}}, \quad G(\xi)=F(\xi)+\frac{\xi}{B_{1}} \int_{-\infty}^{\xi} d \xi^{\prime} \xi^{\prime} F\left(\xi^{\prime}\right) .
$$

2.2.4. Bounds from Realizability Constraints. Using the realizability constraint $Q \in L^{1}\left(R^{1}\right), Q \geq 0$, we can show that

$$
Q=Q_{s}
$$

and

$$
\lim _{\xi \rightarrow+\infty} \xi^{-2} \mathrm{e}^{\Lambda} F(\xi)=0
$$

Indeed, starting from $Q=Q_{s}+C_{1} Q_{1}+C_{2} Q_{2}$, we have:

1. $Q_{s} \in L^{1}\left(R^{1}\right), Q_{2} \in L^{1}\left(R^{1}\right)$, but $Q_{1} \notin L^{1}\left(R^{1}\right)$. Therefore $C_{1}$ must be zero.

2. As $|\xi| \rightarrow+\infty,\left|Q_{2}\right|>\left|Q_{s}\right|$, but $Q_{2}>0$ as $\xi \rightarrow-\infty$, and $Q_{2}<0$ as $\xi \rightarrow+\infty$.

Therefore $C_{2}$ must be zero.

3. As $\xi \rightarrow+\infty, Q_{s} \geq 0$, iff (46) holds.

These statements can be established by evaluating $Q_{2}, Q_{s}$ using Laplace's method. We arrive at:

$$
Q(\xi) \sim \begin{cases}|\xi|^{-3} \int_{-\infty}^{\xi} \xi^{\prime} F\left(\xi^{\prime}\right) d \xi^{\prime} & \text { as } \xi \rightarrow-\infty \\ C_{+} \xi \mathrm{e}^{-\Lambda} & \text { as } \xi \rightarrow+\infty\end{cases}
$$

Once again, we obtain (38). Furthermore we get $\beta=1$ in (30). 
2.2.5. The Exponent 7/2. Let $W\left(s, \xi_{+}, \xi_{-}, x, t\right)$ be the PDF of

$$
(u(y+x, t)-u(y-x, t), \xi(y+x, t), \xi(y-x, t)),
$$

condition on $y$ being a shock location. Then

$$
V_{ \pm}\left(s, \xi_{ \pm}, t\right)=\int_{R^{1}} W\left(s, \xi_{+}, \xi_{-}, 0, t\right) d \xi_{\mp}
$$

$W$ satisfies an equation of the form [35]

$$
\frac{\partial W}{\partial t}=\mathcal{O} W+S
$$

where $\mathcal{O}$ is a differential operator in $x, s, \xi_{ \pm}, S$ is a source term accounting for shock creation and collision. Information on the source term $S$ can be obtained using local analysis around shock creation and collision points. Upon using this information in (48), we get:

$$
F(\xi) \sim C|\xi|^{-5 / 2} \quad \text { as } \xi \rightarrow-\infty
$$

Therefore

$$
Q(\xi) \sim|\xi|^{-3} \int_{-\infty}^{\xi} \xi^{\prime} F\left(\xi^{\prime}\right) d \xi^{\prime}=C_{-}|\xi|^{-7 / 2} \quad \text { as } \xi \rightarrow-\infty
$$

which confirms the prediction of [29].

The analysis in [35] that accomplishes this last step is quite involved. The result of that is a confirmation of the geometric picture proposed in [29], namely the leading order contribution to the left tail of $Q$ comes from the boundary of the set of the shocks, here the points of shock creation. This geometric picture may have interesting consequences in higher dimension. The analysis in [35] is also a success in working with the master equation without making closure assumptions. In a sense closure in [35] is achieved through dimension reduction. The PDF of $\xi$ is first expressed in terms of the lower dimensional dissipative structures, here the shocks. The PDF for the shocks and the shock environment is further expressed in terms of the singular structures on the shocks, namely the points of shock creation and collision, which are then amenable to local analysis. Clearly this approach should be applicable to high dimensions and should yield more interesting results.

As we mentioned earlier, the value of $\alpha$ has been a point of controversy for sometime. Using operator product expansion, Polyakov [90] suggested the value $\alpha=\frac{5}{2}$. Boldyrev [11] extended Polyakov's analysis and suggested that $\alpha$ should be in the interval [2,3]. The value of $\alpha=3$ was obtained by Gotoh and Kraichnan [52] who argued that the dissipation term can be neglected in the tails of the PDF. Other values of $\alpha$ are proposed in [12]. E, Khanin, Mazel and Sinai [29] suggested the value $\alpha=\frac{7}{2}$ by arguing that the dominant contribution comes from the region near the points of shock creation. Very strong analytical support to this result was given in $[\mathbf{3 4}, 35]$. Numerical verification was hindered by the fact that most numerical schemes contain too much numerical viscosity which pollutes the tails of the PDF. Using an innovative Lagrangian approach, Bec was finally able to obtain clean numerical results which give overwhelming support to the value $\alpha=\frac{7}{2}[7,6]$. However, we should remark that even though the prediction for the value of $\alpha$ in [90] is likely to be incorrect, the master equation approach initiated there may have strong impact in the field of turbulence theory. 
2.3. Stochastic Hamilton-Jacobi Equation in High Dimensions. Here we briefly summarize the work of Iturriaga and Khanin [55] which extends some of the results in [30] to high dimensions. The most natural setting is in the context of stochastically forced Hamilton-Jacobi equations on a $d$-dimensional torus $\mathbb{T}^{d}=$ $\mathbb{R}^{d} / \mathbb{Z}^{d}$ :

$$
\varphi_{t}+\frac{1}{2}|\nabla \varphi|^{2}+F^{\omega}(\mathbf{x}, t)=0
$$

where $F^{\omega}(\mathbf{x}, t)=\sum_{k=1}^{N} F_{k}(\mathbf{x}) \dot{W}_{k}(t)$, and $\left\{\dot{W}_{k}(t)\right\}$ are independent white-noises. If we let $\mathbf{u}=\nabla \varphi$, then formally we obtain the $d$-dimensional Burgers system for $\mathbf{u}$ :

$$
\mathbf{u}_{t}+(\mathbf{u} \cdot \nabla) \mathbf{u}+\nabla F^{\omega}(\mathbf{x}, t)=0
$$

This connection can be made rigorous if we add a viscous term. However this only gives irrotational solutions of (53). In principle (53) can have solutions which are not in the form of $\mathbf{u}=\nabla \varphi$ and these solutions may have entirely different properties.

The proper notion of weak solutions is that of the viscosity solution which can also be constructed via the variational principle [75]:

$$
\varphi(\mathbf{x}, t)=\inf _{\gamma(t)=\mathbf{x}}\left(\varphi(\gamma(0), 0)+\mathcal{A}_{0, t}^{\omega}(\gamma)\right)
$$

where

$$
\mathcal{A}_{s, t}^{\omega}=\int_{s}^{t}\left(\frac{1}{2} \dot{\gamma}(\tau)^{2}-F^{\omega}(\gamma(\tau), \tau)\right) d \tau
$$

and the infimum is taken overall absolutely continuous curves $\gamma$ on $[0, t]$ such that $\gamma(t)=\mathbf{x}$. Here as in $\S 2.1$ we have restricted our attention to solutions that satisfy $\int_{\mathbb{T}^{d}} \mathbf{u}(\mathbf{x}, t) d x=0$, but extension to the general case of $\int_{\mathbb{T}^{d}} \mathbf{u}(\mathbf{x}, t) d x=\mathbf{b} \neq 0$ is straightforward (see [55]).

As in the one dimensional case, one can define the notion of OSMs and TSMs (also called global minimizers). The existence of a unique invariant measure can be proved by studying OSMs (see [55]).

The next natural question is the uniqueness and hyperbolicity of the TSM. In this context, we have

THEOREM 9. [55]. Assume that the mapping

$$
\left(F_{1}(\mathbf{x}), \cdots F_{N}(\mathbf{x})\right): \mathbb{T}^{d} \rightarrow \mathbb{R}^{N}
$$

is an embedding. Then for almost all $\omega$ there exists a unique two-sided minimizer for the action $\mathcal{A}^{\omega}$.

The issue of hyperbolicity still remains open.

This theory is intimately related to the theory of homogenization for HamiltonJacobi equation $[\mathbf{7 6}, \mathbf{2 8}, \mathbf{4 0}]$, especially the connection between the stationary solutions to the Hamilton-Jacobi equation and minimizers of the Lagrangian systems (the Aubry-Mather theory). In the deterministic context, such a connection has been exploited in $[27,42,56]$.

\section{Stochastic Passive Scalar Equation}

Consider the transport equation for the scalar field $\theta^{\kappa}(\mathbf{x}, t)$ in $\mathbb{R}^{d}$ :

$$
\frac{\partial \theta^{\kappa}}{\partial t}+(\mathbf{u}(\mathbf{x}, t) \cdot \nabla) \theta^{\kappa}=\kappa \Delta \theta^{\kappa}
$$


where $\mathbf{u}$ is a given (turbulent) velocity field. (54) is the basic equation describing the turbulent transport of a scalar field. Even though this problem is linear, it shares many of the complexities of fully developed turbulence. In the past several years, remarkable progress has been made on the understanding of the structures of such scalar fields $[\mathbf{2}, \mathbf{1 9}, \mathbf{5 0}, \mathbf{5 1}, \mathbf{9 2}]$. Much of this is based on sophisticated fieldtheoretic techniques. Here we will discuss the mathematical work on this problem, drawing mainly from the work in [36].

We will be interested in $\theta^{\kappa}$ in the limit as $\kappa \rightarrow 0$. It is known from classical results that if $\mathbf{u}$ is Lipschitz continuous in $\mathbf{x}$, then as $\kappa \rightarrow 0, \theta^{\kappa}$ converges to $\theta$, the solution of

$$
\frac{\partial \theta}{\partial t}+(\mathbf{u}(\mathbf{x}, t) \cdot \nabla) \theta=0
$$

Furthermore, we can solve this PDE by the method of characteristics. If we define $\left\{\varphi_{s, t}(\mathbf{x})\right\}$ as the flow generated by the velocity field $\mathbf{u}$, satisfying the ordinary differential equations (ODEs)

$$
\frac{d \varphi_{s, t}(\mathbf{x})}{d t}=\mathbf{u}\left(\varphi_{s, t}(\mathbf{x}), t\right), \quad \varphi_{s, s}(\mathbf{x})=\mathbf{x}
$$

for $s<t$, then the solution of the transport equation in (55) for the initial condition $\theta^{\kappa}(\mathbf{x}, 0)=\theta_{0}(\mathbf{x})$ is given by

$$
\theta(\mathbf{x}, t)=\theta_{0}\left(\boldsymbol{\varphi}_{0, t}^{-1}(\mathbf{x})\right)=\theta_{0}\left(\boldsymbol{\varphi}_{t, 0}(\mathbf{x})\right)
$$

This classical scenario breaks down when $\mathbf{u}$ fails to be Lipschitz continuous in $\mathbf{x}$, which is precisely the case for fully developed turbulent velocity fields. In this case Kolmogorov's theory of turbulent flows suggests that $\mathbf{u}$ is only Hölder continuous with an exponent roughly equal to $\frac{1}{3}$ for $d=3$. In such a situation the solution of the ODEs in (56) may fail to be unique, and we then have to consider probability distributions on the set of solutions in order to solve the transport equation in (55). In terms of the ODE (56), this means that we have to consider the notion of generalized flows $[\mathbf{1 5}, \mathbf{1 6}, \mathbf{3 6}, \mathbf{3 8}]$.

When the regularity condition on $\mathbf{u}$ fails, it is natural to consider the limit of physical regularizations. There are at least two natural ways to regularize (56) or (55). The first is to add diffusion:

$$
d \varphi_{s, t}^{\kappa}(\mathbf{x})=\mathbf{u}\left(\varphi_{s, t}^{\kappa}(\mathbf{x}), t\right) d t+\sqrt{2 \kappa} d \boldsymbol{\beta}(t),
$$

and consider the limit as $\kappa \rightarrow 0$. We will call this the $\kappa$-limit. For $\kappa>0$, the diffusion is non-degenerate, and has a unique solution according to Stroock and Varadhan [96]. The second is to smooth out the velocity field. Let $\psi_{\varepsilon}$ be defined as $\psi_{\varepsilon}(\mathbf{x})=\varepsilon^{-d} \psi(\mathbf{x} / \varepsilon)$, where $\psi$ is a standard mollifier: $\psi \geq 0, \int_{\mathbb{R}^{d}} \psi d x=1, \psi$ decays fast at infinity. Let $\mathbf{u}^{\varepsilon}=\mathbf{u} \star \psi_{\varepsilon}$ and consider

$$
\frac{d \varphi_{s, t}^{\varepsilon}(\mathbf{x})}{d t}=\mathbf{u}^{\varepsilon}\left(\varphi_{s, t}^{\varepsilon}(\mathbf{x}), t\right)
$$

in the limit as $\varepsilon \rightarrow 0$. We will call this the $\varepsilon$-limit. Physically $\kappa$ plays the role of molecular diffusivity, $\varepsilon$ can be thought of as a crude model of the viscous cutoff scale. In terms of the Prandtl number $\operatorname{Pr}=\frac{\varepsilon}{\kappa}$ the $\kappa$-limit corresponds to the situation when $\operatorname{Pr} \rightarrow 0$, whereas the $\varepsilon$-limit corresponds to the situation when $\operatorname{Pr} \rightarrow \infty$. 
There are two ways to think about probability distributions on the solutions of the ODEs in (56). We can either think of it as probability measures on the pathspace (functions of $t$ ) supported by paths which are solutions of (56), or we can think of it as transition probability at time $t$ if the starting position at time $s$ is $\mathbf{x}$. In the classical situation when $\mathbf{u}$ is Lipschitz continuous, this transition probability degenerates to a point mass centered at the unique solution of (56). When Lipschitz condition fails, this transition probability may be non-degenerate and the system in (56) is intrinsically stochastic. We will adopt this latter approach and use this transition probability to define the "generalized flows".

Definition. $[\mathbf{1 5}, \mathbf{1 6}, \mathbf{3 8}, \mathbf{7 2}]$ Let $\{g(\mathbf{x}, s \mid d \mathbf{y}, t)\}$ be a family of probability measures, which are continuous in $(\mathbf{x}, s, t)$, and satisfy

1.

$$
\begin{aligned}
\int_{s}^{t} \int_{\mathbb{R}^{d}}\left\{\frac{\partial \phi}{\partial t}(\mathbf{y}, \tau)+\mathbf{u}(\mathbf{y}, \tau) \cdot \nabla_{y} \phi(\mathbf{y}, \tau)\right\} g(\mathbf{x}, s \mid d \mathbf{y}, \tau) d \tau \\
\\
-\int \phi(\mathbf{y}, t) g(\mathbf{x}, s \mid d \mathbf{y}, t)+\phi(\mathbf{x}, s)=0
\end{aligned}
$$

for all test functions $\phi \in C^{\infty}\left([0, \infty) \times \mathbb{R}^{d}\right.$.

2. The Chapman-Kolmogorov equation

$$
g(\mathbf{x}, s \mid d \mathbf{y}, t)=\int_{\mathbb{R}^{d}} g(\mathbf{x}, s \mid d \mathbf{z}, \tau) g(\mathbf{z}, \tau \mid d \mathbf{y}, t)
$$

for all $\tau \in[s, t]$.

Then $\{g(\mathbf{x}, s \mid d \mathbf{y}, t)\}$ is called a generalized flow.

This definition only takes into account the so-called separable generalized flows. Non-separable generalized flows are considered in [38].

Before proceeding further, we relate the regularized flows in (58), (59) to the solutions of the transport equations. Consider the $\kappa$-regularization first. It is convenient to introduce the backward transition probability

$$
g^{\kappa}(\mathbf{x}, t \mid d \mathbf{y}, s)=\mathbf{E}_{\boldsymbol{\beta}^{\delta}}\left(\mathbf{y}-\varphi_{t, s}^{\kappa}(\mathbf{x})\right) d \mathbf{y}, \quad s<t,
$$

where the expectation is taken with respect to $\boldsymbol{\beta}(t)$, and $\boldsymbol{\varphi}_{t, s}^{\kappa}(\mathbf{x})$ is the flow inverse to $\varphi_{s, t}^{\kappa}(\mathbf{x})$ defined in (58) (i.e. $\varphi_{s, t}^{\kappa}(\mathbf{x})$ is the forward flow and $\varphi_{t, s}^{\kappa}(\mathbf{x})$ is the backward flow). The action of $g^{\kappa}$ generates a semi-group of transformation

$$
S_{t, s}^{\kappa} \psi(\mathbf{x})=\int_{\mathbb{R}^{d}} \psi(\mathbf{y}) g^{\kappa}(\mathbf{x}, t \mid d \mathbf{y}, s),
$$

for all test functions $\psi \cdot \theta^{\kappa}(\mathbf{x}, t)=S_{t, s}^{\kappa} \psi(\mathbf{x})$ solve the transport equation in (54) for the initial condition $\theta^{\kappa}(\mathbf{x}, s)=\psi(\mathbf{x})$. Similarly, for the flow in (59), define

$$
S_{t, s}^{\varepsilon} \psi(\mathbf{x})=\psi\left(\varphi_{t, s}^{\varepsilon}(\mathbf{x})\right), \quad s<t .
$$

$\theta^{\varepsilon}(\mathbf{x}, t)=S_{t, s}^{\varepsilon} \psi(\mathbf{x})$ solves the transport equation

$$
\frac{\partial \theta^{\varepsilon}}{\partial t}+\left(\mathbf{u}^{\varepsilon}(\mathbf{x}, t) \cdot \nabla\right) \theta^{\varepsilon}=0
$$

with initial condition $\theta(\mathbf{x}, s)=\psi(\mathbf{x})$. Similar definitions can be given for forward flows but we will restrict attention to the backward ones since we are primarily interested in scalar transport. The results given below generalize trivially to forward flows. 


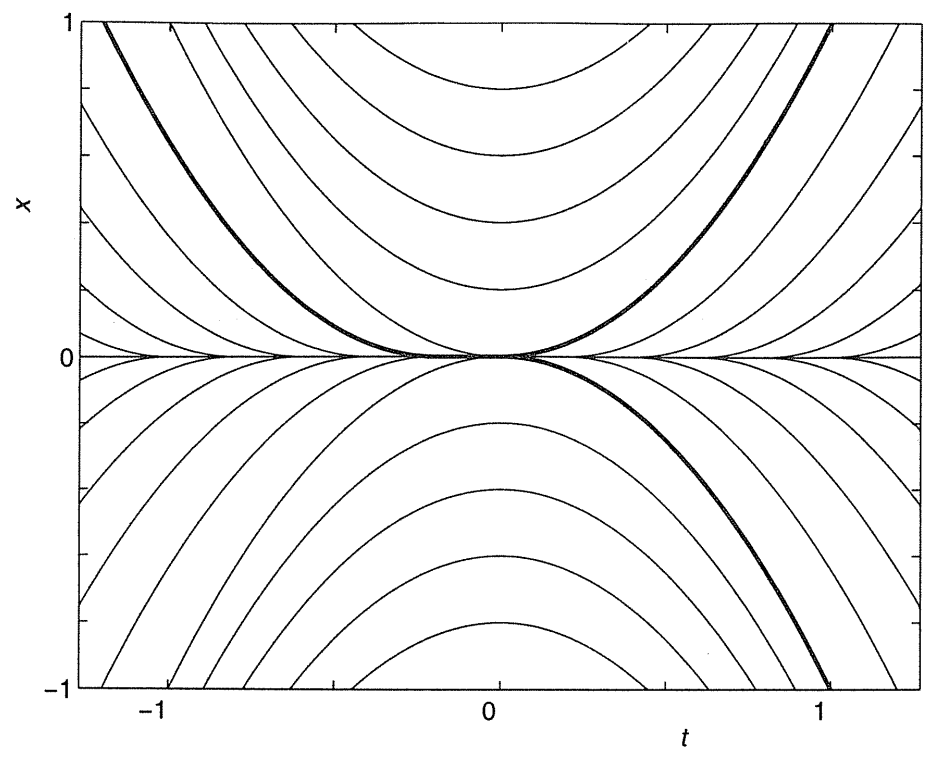

FiguRE 1. In the $\kappa$-limit, the particle coming from the left along the highlighted trajectory has equal probablity to take the two highlighted trajectories on the right.

3.1. A Simple Example of Generalized Flows. Let us consider the following example in the $\kappa$-limit:

$$
d x=\operatorname{sgn}(x t) x^{\frac{1}{2}} d t+\sqrt{2 \kappa} d \beta(t), \quad x(0)=y
$$

To avoid the discontinuity at $t=0$, we replace the drift term by $2 t$ for $x>\frac{1}{4} t^{2}$ and by $-2 t$ for $x<-\frac{1}{4} t^{2}$. We will denote the solutions as $x_{t}^{\kappa}(y)$. It is easy to see that as $\kappa \rightarrow 0, x_{t}^{\kappa}(y)$ converges to the unique solution of $\frac{d x}{d t}=x^{\frac{1}{2}}$ if $y \neq 0$, and when $y=0, x_{t}^{\kappa}(y)$ converges to the random process that takes the extreme trajectories $x_{1}(t)=\frac{1}{4} t^{2}$ and $x_{2}(t)=-\frac{1}{4} t^{2}$, with equal probability (see Figure 1 ).

A slightly more interesting example is described in Figure 2. Here the velocity field is time-dependent and fails to be Lipschitz continuous at the nodes. In Figure 2 , instead of plotting $u$, we plotted the possible trajectories that are solutions of the ODE $\frac{d x}{d t}=u$. It is easy to check that in the $\kappa$-limit, we obtain a symmetric random walk on the separatrices, which are highlighted in Figure 2 by heavy lines.

3.2. Delta-correlated Gaussian Velocity Fields. In [63] Kraichnan introduced a simple model of passive scalar transport by considering the advection by a Gaussian, spatially non-smooth, Kolmogorov-like and white-in-time velocity field. This class of velocity fiels is now referred to as the Kraichnan model. Definitive progress has been made in the last several years on the understanding of the structure functions and intermittency associated with the Kraichnan model $[8,19,50,51,78,92]$. Much of these are done using fairly sophisticated perturbative field theory. We will first present the mathematical framework, followed by a summary of the statistical theory. We consider a generalization of the Kraichnan model introduced in [51] that takes into account compressibility effects (see also [72]). The velocity field $\mathbf{u}$ is assumed to be a statistically homogeneous, isotropic 


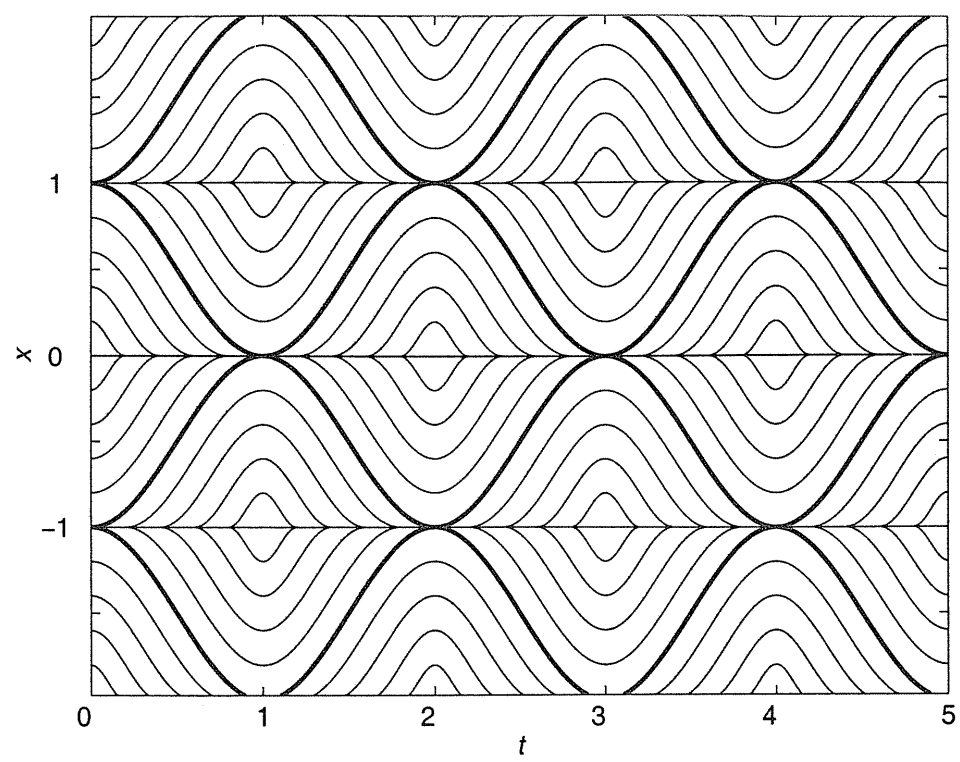

FIGURE 2. In the $\kappa$-limit, a tracer particle performs a symmetric random walk on the network formed by the highlighted trajectories.

and stationary Gaussian field with mean zero and covariance

$$
\mathbb{E} u_{\alpha}(\mathbf{x}, t) u_{\beta}(\mathbf{y}, s)=\left(C_{0} \delta_{\alpha \beta}-c_{\alpha \beta}(\mathbf{x}-\mathbf{y})\right) \delta(t-s) .
$$

We assume that $\mathbf{u}$ has a correlation length $\ell_{0}$, i.e. the covariance in (65) decays fast for $|\mathbf{x}-\mathbf{y}|>\ell_{0}$. Consequently $c_{\alpha \beta}(\mathbf{x}) \rightarrow C_{0} \delta_{\alpha \beta}$ as $|\mathbf{x}| / \ell_{0} \rightarrow \infty$. On the other hand, we will be mainly interested in small scale phenomena for which $|\mathbf{x}| \ll \ell_{0}$. In this range, we take $c_{\alpha \beta}(\mathbf{x})=d_{\alpha \beta}(\mathbf{x})+O\left(|\mathbf{x}|^{2} / \ell_{0}^{2}\right)$ with

$$
d_{\alpha \beta}(\mathbf{x})=A d_{\alpha \beta}^{P}(\mathbf{x})+B d_{\alpha \beta}^{S}(\mathbf{x})
$$

and

$$
\begin{aligned}
& d_{\alpha \beta}^{P}(\mathbf{x})=D\left(\delta_{\alpha \beta}+\xi \frac{x_{\alpha} x_{\beta}}{|\mathbf{x}|^{2}}\right)|\mathbf{x}|^{\xi} \\
& d_{\alpha \beta}^{S}(\mathbf{x})=D\left((d+\xi-1) \delta_{\alpha \beta}-\xi \frac{x_{\alpha} x_{\beta}}{|\mathbf{x}|^{2}}\right)|\mathbf{x}|^{\xi}
\end{aligned}
$$

Here $D$ is just a parameter. The parameters $A$ and $B$ measure the divergence and rotation of the field $\mathbf{u}$. $A=0$ corresponds to incompressible fields with $\nabla \cdot \mathbf{u}=$ 0 . $B=0$ corresponds to irrotational fields with $\nabla \times \mathfrak{u}=0$. The parameter $\xi$ measures the spatial regularity of u. For $\xi \in(0,2)$, the local characteristic of u fails to be twice differentiable. This means roughly that $\mathbf{u}$ is spatially only Hölder continuous with exponent $\frac{\xi}{2}$. We call such velocity fields Kolmogorov-like. This spatial behavior has important consequences on both the transport equation (55) and the systems of ODEs (56).

Let $\mathcal{S}^{2}=A+(d-1) B, \mathcal{C}^{2}=A, \mathcal{P}=\mathcal{C}^{2} / \mathcal{S}^{2} . \mathcal{P} \in[0,1]$ is a measure of the degree of compressibility of $\mathbf{u}$.

The following result is a classification of the different regimes we obtain when we consider the $\kappa$ - and $\varepsilon$-limits. 
THEOREM 10. There exist three different regimes:

1. $\mathcal{P} \geq d / \xi^{2}$, the strongly compressible regime. In this case, there exists a family of random maps $\left\{\varphi_{t, s}^{\omega}(\mathbf{x})\right\}$, such that for all smooth test functions $\psi$ and for all $(s, t, \mathbf{x}), s<t$,

$$
\mathbf{E}\left(S_{t, s}^{\omega, \kappa} \psi(\mathbf{x})-\psi\left(\varphi_{t, s}^{\omega}(\mathbf{x})\right)\right)^{2} \rightarrow 0
$$

as $\kappa \rightarrow 0$, and

$$
\mathbf{E}\left(\psi\left(\boldsymbol{\varphi}_{t, s}^{\omega, \varepsilon}(\mathbf{x})\right)-\boldsymbol{\psi}\left(\boldsymbol{\varphi}_{t, s}^{\omega}(\mathbf{x})\right)\right)^{2} \rightarrow 0,
$$

as $\varepsilon \rightarrow 0$. Moreover, the limiting flow $\left\{\varphi_{t, s}^{\omega}(\mathbf{x})\right\}$ coalesces in the sense that for almost all $(t, \mathbf{x}, \mathbf{y}), \mathbf{x} \neq \mathbf{y}$, we can define a time $\tau$ such that $-\infty<\tau<t$ a.s. and

$$
\varphi_{t, s}^{\omega}(\mathbf{x})=\varphi_{t, s}^{\omega}(\mathbf{y}) \quad \text { for } s \leq \tau .
$$

2. $\mathcal{P} \leq(d+\xi-2) / 2 \xi$, the weakly compressible regime. In this case there exists a random family of generalized flows $g_{\omega}(\mathbf{x}, t \mid d \mathbf{y}, s)$, such that for all test function $\psi$,

$$
S_{t, s}^{\omega} \psi(\mathbf{x})=\int_{\mathbb{R}^{d}} \psi(\mathbf{y}) g_{\omega}(\mathbf{x}, t \mid d \mathbf{y}, s)
$$

satisfies

$$
\mathbf{E}\left(S_{t, s}^{\omega, \kappa} \psi(\mathbf{x})-S_{t, s}^{\omega} \psi(\mathbf{x})\right)^{2} \rightarrow 0
$$

as $\kappa \rightarrow 0$ for all $(s, t, \mathbf{x}), s<t$, and

$$
\mathbb{E}\left(\int_{\mathbb{R}^{d}} \eta(\mathbf{x})\left(\psi\left(\varphi_{t, s}^{\omega, \varepsilon}(\mathbf{x})\right)-S_{t, s}^{\omega} \psi(\mathbf{x})\right) d x\right)^{2} \rightarrow 0
$$

as $\varepsilon \rightarrow 0$ for all $(s, t), s<t$, and for all test functions $\eta$. Moreover, $g_{\omega}(\mathbf{x}, t \mid d \mathbf{y}, s)$ is non-degenerate in the sense that there exists a $\psi$, such that there exists a $\psi$, such that

$$
S_{t, s}^{\omega} \psi^{2}(\mathbf{x})-\left(S_{t, s}^{\omega} \psi(\mathbf{x})\right)^{2}>0 \quad \text { a.s. }
$$

3. $(d+\xi-2) / 2 \xi<P<d / \xi^{2}$, the intermediate regime. In this case there exists a random family of generalized flows $g_{\omega}(\mathbf{x}, t \mid d \mathbf{y}, s)$, such that for all test function $\psi$ and for all $(s, t, \mathbf{x}), s<t$,

$$
\mathbb{E}\left(S_{t, s}^{\omega, \kappa} \psi(\mathbf{x})-S_{s, t}^{\omega} \psi(\mathbf{x})\right)^{2} \rightarrow 0
$$

as $\kappa \rightarrow 0$. In the $\varepsilon$-limit, the flows $\varphi_{t, s}^{\omega, \varepsilon}(\mathbf{x})$ converges in the sense of distributions, i.e. there exists a family of probability densities $\left\{G_{n}\left(\mathbf{x}_{1}, \ldots, \mathbf{x}_{n}, t \mid \mathbf{y}_{1}, \ldots, \mathbf{y}_{n}, s\right) d \mathbf{y}_{1} \cdots d \mathbf{y}\right.$ $n=1,2, \ldots$, such that

$$
\begin{array}{r}
\mathbb{E} \psi\left(\varphi_{t, s}^{\omega, \varepsilon}\left(\mathbf{x}_{1}\right), \cdots, \varphi_{t, s}^{\omega, \varepsilon}\left(\mathbf{x}_{n}\right)\right) \rightarrow \int_{\mathbb{R}^{d} \times \cdots \times \mathbb{R}^{d}} \psi\left(\mathbf{y}_{1}, \cdots, \mathbf{y}_{n}\right) \\
\times G_{n}\left(\mathbf{x}_{1}, \cdots, \mathbf{x}_{n}, t \mid \mathbf{y}_{1}, \ldots, \mathbf{y}_{n}, s\right) d \mathbf{y}_{1} \cdots d \mathbf{y}_{n},
\end{array}
$$

as $\varepsilon \rightarrow 0$ for any continuous function $\psi$ with compact support. Furthermore, there is coalesces in the $\varepsilon$-limit in the sense that

$$
\begin{aligned}
G_{2}\left(\mathbf{x}_{1}, \mathbf{x}_{2}, t \mid \mathbf{y}_{1}, \mathbf{y}_{2}, s\right) & =\tilde{G}_{2}\left(\mathbf{x}_{1}, \mathbf{x}_{2}, t \mid \mathbf{y}_{1}, \mathbf{y}_{2}, s\right) \\
+ & A\left(y_{1}, \mathbf{x}_{1}, \mathbf{x}_{2}, t, s\right) \delta\left(\mathbf{y}_{1}-\mathbf{y}_{2}\right),
\end{aligned}
$$


with $A>0$ when $t>s$. Here $\tilde{G}_{2}$ is the absolutely continuous part of $G_{2}$ with respect to the Lebesgue measure. Similar statements hold for the other $G_{n}$ 's. In particular, the $\left\{G_{n}\right\}$ 's differ from the moments of the $\kappa$-limit $g_{\omega}$ defined in (75).

The family of probability densities $\left\{G_{n}\right\}$ are consistent in the sense of Kolmogorov. Therefore there exists a stochastic flow defined on some probability space with $\left\{G_{n}\right\}$ as its distribution [69].

Rephrasing the content of this result, we have strong convergence to a family of flow maps in the strongly compressible regime for both the $\kappa$-limit and the $\varepsilon$ limit. In the weakly compressible regime, we have strong convergence to a family of generalized flows for the $\kappa$-limit, but weak convergence to the same limit for the $\varepsilon$ regularization. In fact, using the terminology of Young measures [98], the limiting generalized flow $\left\{g_{\omega}(\mathbf{x}, t \mid d \mathbf{y}, s)\right\}$ is nothing but the Young measure for the sequence of oscillating flow maps $\left\{\boldsymbol{\varphi}_{s, t}^{\omega, \varepsilon}(\mathbf{x})\right\}$. Finally, in contrast to what is observed in the other two regimes, the $\varepsilon$-limit and $\kappa$-limit are not the same in the intermediate regime.

Theorem 10 in its present form is found in [36]. Earlier Gawedzki, Vergassola [51] and independently Le Jan and Raimond [72] considered the $\kappa$-limit. In that case there is only the difference of convergence to a family of flow maps in the strongly compressible and intermediate regimes, and convergence to generalized stochastic flows in the weakly compressible regime. Joint limits $\kappa, \varepsilon \rightarrow 0$ were studied in [37] and it was concluded that the limiting behavior depends on a new "turbulent Prandtl number."

¿From Theorem 10, it is natural to define the solution of the transport equation in (55) for the initial condition $\theta_{\omega}(\mathbf{x}, s)=\theta_{0}(\mathbf{x})$ as

$$
\theta_{\omega}(\mathbf{x}, t)=S_{t, s}^{\omega} \theta_{0}(\mathbf{x})=\int_{\mathbb{R}^{d}} \theta_{0}(\mathbf{y}) g_{\omega}(\mathbf{x}, t \mid d \mathbf{y}, s),
$$

for the weakly compressible and the intermediate regimes in the $\kappa$-limit (nondegenerate cases), and as

$$
\theta_{\omega}(\mathbf{x}, t)=\theta_{0}\left(\varphi_{t, s}^{\omega}(\mathbf{x})\right)
$$

for the strongly compressible regime. In the intermediate regime in the $\varepsilon$-limit, it makes sense to look at the limiting moments of $\theta_{\omega}^{\varepsilon}(\mathrm{x}, t)$ since we have as $\varepsilon \rightarrow 0$

$$
\begin{aligned}
& \mathbb{E}\left(\theta_{\omega}^{\varepsilon}\left(\mathbf{x}_{1}, t\right) \cdots \theta_{\omega}^{\varepsilon}\left(\mathbf{x}_{n}, t\right)\right) \rightarrow \int_{\mathbb{R}^{d} \times \cdots \times \mathbb{R}^{d}} \theta_{0}\left(\mathbf{y}_{1}\right) \cdots \theta_{0}\left(\mathbf{y}_{n}\right) \\
& \times G_{n}\left(\mathbf{x}_{1}, \cdots, \mathbf{x}_{n}, t \mid \mathbf{y}_{1}, \ldots, \mathbf{y}_{n}, s\right) d \mathbf{y}_{1} \cdots d \mathbf{y}_{n} .
\end{aligned}
$$

An outline of the proof of Thoerem 10 is as follows. Define $P(\rho \mid r, s)$ through $\varepsilon$-regularization as

$$
\int_{0}^{\infty} \eta(r) P(\rho \mid r, s-t) d r=\lim _{\varepsilon \rightarrow 0} \mathbb{E} \eta\left(\left|\varphi_{t, s}^{\omega, \varepsilon}(\mathbf{y})-\varphi_{t, s}^{\omega, \varepsilon}(\mathbf{z})\right|\right)
$$

where $\eta$ is a test function, and similarly through $\kappa$-regularization. Here $\rho=|\mathbf{y}-\mathbf{z}|$ and $s<t$. $P(\rho \mid r, s)$ can be thought of as the probability density that two particles have distance $r$ at time $s<t$ if their final distance is $\rho$ at time $t$. For the Kraichnan model, $P$ satisfies the backward equation

$$
-\frac{\partial P}{\partial s}=-\frac{\partial}{\partial r}(b(r) P)+\frac{\partial^{2}}{\partial r^{2}}(a(r) P)
$$


for the final condition $\lim _{s \rightarrow 0-} P(\rho \mid r, s)=\delta(r-\rho)$, and $a(r), b(r)$ have the behavior

$$
\begin{gathered}
a(r)=D\left(\mathcal{S}^{2}+\xi \mathcal{C}^{2}\right) r^{\xi}+O\left(r^{2} / \ell_{0}^{2}\right), \\
b(r)=D\left((d-1+\xi) \mathcal{S}^{2}-\xi \mathcal{C}^{2}\right) r^{\xi-1}+O\left(r / \ell_{0}^{2}\right) .
\end{gathered}
$$

For $r \gg \ell_{0}, a(r)$ tends to $C_{0}$, and $b(r)$ tends to $C_{0}(d-1) / r$. The equation (82) then reduces to a diffusion equation with constant coefficients. At $r=0$ the equation (82) is singular. The proof of Theorem 10 is essentially reduced to the study of this singular diffusion equation.

The behavior of the structure functions in these different limits is discussed in $[36,37,51]$.

3.3. Invariant Measures. Here we study the existence of an invariant measure for the transport equation with appropriate forcing. We restrict attention to the non-degenerate cases which include the weakly compressible regime and the intermediate regime in the $\kappa$-limit. In these regimes the limiting generalized flow is non-degenerate and hence behaves as a non-trivial diffusion process. The consequences of this is that there is a dissipation mechanism present (as we will discuss further in Section 5), and the system losses memory. We show that the anomalous dissipation is strong enough for the forced transport equation to have a unique invariant measure for both the weakly compressible regime and the intermediate regime in the $\kappa$-limit.

Consider (compare with (55))

$$
\frac{\partial \theta}{\partial t}+(\mathbf{u}(\mathbf{x}, t) \cdot \nabla) \theta=b(\mathbf{x}, t) .
$$

where $b$ is a white-noise forcing such that

$$
\mathbb{E} b(\mathbf{x}, t) b(\mathbf{y}, s)=B(|\mathbf{x}-\mathbf{y}|) \delta(t-s) .
$$

$B(r)$ is assumed to be smooth and rapidly decaying to zero for $r \gg L$; $L$ will be referred to as the forcing scale. The solution of (84) for the initial condition $\theta_{\omega}(\mathbf{x}, s)=\theta_{0}(\mathbf{x})$ is understood as

$$
\theta_{\omega}(\mathbf{x}, t)=S_{t, s}^{\omega} \theta_{0}(\mathbf{x})+\int_{s}^{t} S_{t, \tau}^{\omega} b(\mathbf{x}, \tau) d \tau .
$$

Define the product probability space $\left(\Omega_{u} \times \Omega_{b}, \mathcal{F}_{u} \times \mathcal{F}_{b}, \mathcal{P}_{u} \times \mathcal{P}_{b}\right)$, and the shift operator $T_{\tau} \omega(t)=\omega(t+\tau)$, with $\omega=\left(\omega_{u}, \omega_{b}\right)$. We have

THEOREM 11. For $d>2$, in the weakly compressible regime and in the intermediate regime in the $\kappa$-limit, for almost all $\omega$, there exists a unique solution of (84) defined on $\mathbb{R}^{d} \times(-\infty, \infty)$. This solution can be expressed as

$$
\theta_{\omega}^{\star}(\mathbf{x}, t)=\int_{-\infty}^{t} S_{t, s}^{\omega} b(\mathbf{x}, s) d s .
$$

Furthermore the map $\omega \rightarrow \theta_{\omega}^{\star}$ satisfies the invariance property

$$
\theta_{T_{\tau} \omega}^{\star}(\mathbf{x}, t)=\theta_{\omega}^{\star}(\mathbf{x}, t+\tau) .
$$

Theorem 11 is the "one force, one solution" principle articulated in [30], and discussed earlier. Because of the invariance property (88), the map in (87) leads to a natural invariant measure. As a consequence we have 
COROLlary 1. For $d>2$, in the weakly compressible regime and in the intermediate regime in the $\kappa$-limit, there exists a unique invariant measure on $L_{\text {loc }}^{2}\left(\mathbb{R}^{d} \times\right.$ $\Omega)$ for the dynamics defined by (84).

To prove Theorem 11, we verify that the dissipation in the system is strong enough in the sense that

$$
\mathbf{E}\left(\int_{T_{1}}^{T_{2}} \int_{\mathbb{R}^{d}} S_{t, t+s}^{\omega} b(\mathbf{x}, s) d s\right)^{2} \rightarrow 0,
$$

as $T_{1}, T_{2} \rightarrow-\infty$ for fixed $x$ and $t$. The average in (89) is given explicitly by

$$
\int_{T_{1}}^{T_{2}} \int_{0}^{\infty} B(\rho) P(0 \mid \rho, s) d \rho d s
$$

where $P$ satisfies (82). The convergence of the integral in (89) depends on the rate of decay in $|s|$ of $P(0 \mid \rho, s)$. The latter can be estimated by studying the equation in (82) [36], which yields $P(0 \mid \rho, s) \sim C \rho^{\alpha}|s|^{-d / 2}$ with $\alpha=(d-1-\xi(\xi+1) \mathcal{P}) /(1+\xi \mathcal{P})$ for $|s|$ large and $\rho \ll \ell_{0}$. Hence, the integral in $s$ in (90) tends to zero as $T_{1}$, $T_{2} \rightarrow-\infty$ if $d>2$. It follows that the invariant measure in (87) exists provided that $d>2$.

Since no anomalous dissipation is present in the coalescence cases, i.e the strongly compressible regime and the intermediate regime in the $\varepsilon$-limit, no invariant measure for the temperature field exists in these regimes. It makes sense, however, to ask about the existence of an invariant measure for the temperature difference, i.e. to consider

$$
\delta \theta_{\omega}(\mathbf{x}, \mathbf{y}, t)=\int_{T}^{t} S_{t, s}^{\omega}(b(\mathbf{x}, s)-b(\mathbf{y}, s)) d s
$$

in the limit as $T \rightarrow-\infty$. When $\theta_{\omega}^{\star}$ exists, one has

$$
\delta \theta_{\omega}^{\star}(\mathbf{x}, \mathbf{y}, t)=\lim _{T \rightarrow-\infty} \delta \theta_{\omega}(\mathbf{x}, \mathbf{y}, t)=\theta_{\omega}^{\star}(\mathbf{x}, t)-\theta_{\omega}^{\star}(\mathbf{y}, t),
$$

but it is conceivable that $\delta \theta_{\omega}^{\star}$ exists in the coalescence cases even though $\theta_{\omega}^{\star}$ is not defined. The reason is that coalescence of the generalized flow implies that the temperature field flattens with time, which is a dissipation mechanism as far as the temperature difference is concerned. Of course, this effect has to overcome the fluctuations produced by the forcing, and the existence of the limit in (91) will depend on how fast particles coalesce under the flow.

For finite $\ell_{0}$, if we consider two particles separated by a distance much longer than the correlation length $\ell_{0}$, the dynamics of their distance under the flow is governed by the equation in (82) for $r \gg \ell_{0}$, i.e. by a diffusion equation with constant coefficient on the scale of interest. It follows that no tendency of coalescence is observed before the distance becomes smaller than $\ell_{0}$, which does not happen fast enough in order to overcome the the fluctuations produced by the forcing. In other words,

LEMMA 2. In the coalescence cases, for finite $\ell_{0}$, there is no invariant measure with finite energy for the temperature difference.

Therefore it is natural to consider the limit as $l_{0} \rightarrow \infty$. However we have to be careful because the velocity field with the covariance in (65) diverges as $\ell_{0} \rightarrow \infty$. 
The right way to proceed is to consider an alternative velocity $\mathbf{v}$, taken to be Gaussian, white-in-time, but non-homogeneous, with covariance

$$
\begin{aligned}
& \mathbf{E} v_{\alpha}(\mathbf{x}, t) v_{\beta}(\mathbf{y}, s) \\
& =\left(c_{\alpha \beta}(\mathbf{x}-\mathbf{a})+c_{\alpha \beta}(\mathbf{a}-\mathbf{y})-c_{\alpha \beta}(\mathbf{x}-\mathbf{y})\right) \delta(t-s) .
\end{aligned}
$$

For finite $\ell_{0}$, one has $v(\mathbf{x}, t)=u(\mathbf{x}, t)-u(\mathbf{a}, t)$, where $\mathbf{a}$ is arbitrary but fixed. However, $\mathbf{v}$ makes sense in the limit as $\ell_{0} \rightarrow \infty$. Denote by $\vartheta_{\omega}(\mathbf{x}, t)$ the temperature field advected by $\mathbf{v}$, i.e. the solution of the transport equation (84) with $\mathbf{u}$ replaced by $\mathbf{v}$ :

$$
\frac{\partial \vartheta}{\partial t}+(\mathbf{v}(\mathbf{x}, t) \cdot \nabla) \vartheta=b(\mathbf{x}, t)
$$

Restricting to zero initial condition, it follows from the homogeneity of the forcing that the single-time moments of $\theta_{\omega}$ and $\vartheta_{\omega}$ coincide for finite $\ell_{0}$, but in contrast to $\theta_{\omega}, \vartheta_{\omega}$ makes sense as $\ell_{0} \rightarrow \infty$. Furthermore, Theorem 10 can be extended to (94) in the limit as $\ell_{0} \rightarrow \infty$.

Let $\delta \vartheta_{\omega}(\mathbf{x}, \mathbf{y}, t)=\vartheta_{\omega}(\mathbf{x}, t)-\vartheta_{\omega}(\mathbf{y}, t)$ where $\vartheta_{\omega}$ solves the equation in (94). The temperature difference $\delta \vartheta_{\omega}$ satisfies the transport equation

$$
\frac{\partial \delta \vartheta}{\partial t}+\left(\mathbf{v}(\mathbf{x}, t) \cdot \nabla_{\mathbf{x}}+\mathbf{v}(\mathbf{y}, t) \cdot \nabla_{\mathbf{y}}\right) \delta \vartheta=b(\mathbf{x}, t)-b(\mathbf{y}, t)
$$

We have

THEOREM 12. In the limit as $\ell_{0} \rightarrow \infty$, for almost all $\omega$, in the strongly and the weakly compressible regimes, as well as in the intermediate regime if the flow is non-degenerate, there exists a unique solution of (95) defined on $\mathbb{R}^{d} \times(-\infty, \infty)$. This solution can be expressed as

$$
\delta \vartheta_{\omega}^{\star}(\mathbf{x}, \mathbf{y}, t)=\int_{-\infty}^{t} S_{t, s}^{\omega}(b(\mathbf{x}, s)-b(\mathbf{y}, s)) d s
$$

where $S_{s, t}^{\omega}$ is the semi-group for the generalized flow associated with the velocity defined in (93) in the limit as $\ell_{0} \rightarrow \infty$. Furthermore the map $\omega \rightarrow \delta \vartheta_{\omega}^{\star}$ satisfies the invariance property

$$
\delta \vartheta_{T_{\tau} \omega}^{\star}(\mathbf{x}, \mathbf{y}, t)=\delta \vartheta_{\omega}^{\star}(\mathbf{x}, \mathbf{y}, t+\tau)
$$

An immediate consequence of this theorem is

COROLlary 3. In the limit as $\ell_{0} \rightarrow \infty$, in the strongly and the weakly compressible regimes, as well as in the intermediate regime if the flow is non-degenerate, there exists a unique invariant measure on $L_{\text {loc }}^{2}\left(\mathbb{R}^{d} \times \Omega\right)$ for the dynamics defined by (95).

The proof of Theorem 12 proceeds similarly as the proof of Theorem 11. In the non-degenerate cases, one studies the convergence of (compare with (89))

$$
\mathrm{E}\left(\int_{T_{1}}^{T_{2}} \int_{\mathbb{R}^{d}} S_{t, t+s}^{\omega}(b(\mathbf{x}, s)-b(\mathbf{y}, s)) d s\right)^{2} \rightarrow 0,
$$

as $T_{1}, T_{2} \rightarrow-\infty$ for fixed $x$ and $t$. The average in (98) can be expressed in terms of $P$, and it can be shown that the expression in (98) converges as $T_{1}, T_{2} \rightarrow-\infty$ 
in the non-degenerate cases. In the strongly compressible regimes, because of the existence of a flow of maps, (98) is replaced by

$$
\mathbf{E}\left(\int_{T_{1}}^{T_{2}}\left(b\left(\varphi_{t, s}^{\omega}(\mathbf{x}), s\right)-b\left(\varphi_{t, s}^{\omega}(\mathbf{y}), s\right)\right) d s\right)^{2}
$$

This average can again be expressed in terms of $P$, and it can be shown that the convergence of the time integral in (99) depends on the rate at $P$ looses mass at $r=0+$ (i.e. the rate at which particles coalesce). The analysis of the equation in (82) shows that the process is fast enough in order that the integral over $s$ in (99) tends to zero as $T_{1}, T_{2} \rightarrow-\infty$ in the strongly compressible regime. In contrast, the equivalent of (99) in the intermediate regime in the $\varepsilon$-limit can be shown to diverge as $T_{1}, T_{2} \rightarrow-\infty$.

3.4. Correlation Functions and Zero Modes. In this section, we briefly summarize the perturbation theory developed in $[\mathbf{5 0 , 1 9 ]}$ on the behavior of the $n$-point correlation functions. These results rely on the fact that a closed set of equations for the single time correlation functions can be derived for the Kraichnan model. We will restrict ourselves to the incompressible case when $A=0$.

Consider

$$
F_{n}\left(\mathbf{x}_{1}, \cdots, \mathbf{x}_{n}, t\right)=\left\langle\theta\left(\mathbf{x}_{1}, t\right) \cdots\left(\theta\left(\mathbf{x}_{n}, t\right)\right\rangle\right.
$$

where $\theta$ satisfies (84) $F_{2 n-1}=0$ by summetry. $F_{2 n}$ satisfies

$$
\begin{aligned}
& \frac{\partial}{\partial t} F_{2 n}\left(\mathbf{x}_{1}, \cdots, \mathbf{x}_{2 n}, t\right)= \sum_{j, k=1}^{2 n} C_{\alpha \beta}\left(\mathbf{x}_{j}-\mathbf{x}_{k}\right) \nabla_{\alpha}^{j} \nabla_{\beta}^{k} F_{2 n}\left(\mathbf{x}_{1}, \cdots, \mathbf{x}_{2 n}, t\right) \\
&+2 \sum_{\substack{j, k=1 \\
j<k}}^{2 n} B\left(\left|\mathbf{x}_{j}-\mathbf{x}_{k}\right|\right) F_{2 n-2}\left(\mathbf{x}_{1}, \cdots, \mathbf{x}_{2 n}, t\right) . \\
& \hat{j} \hat{k}
\end{aligned}
$$

At statistical steady state, these equations reduce to

$$
\begin{aligned}
& \sum_{j, k=1}^{2 n} C_{\alpha \beta}\left(\mathbf{x}_{j}-\mathbf{x}_{k}\right) \nabla_{\alpha}^{j} \nabla_{\beta}^{k} F_{2 n}\left(\mathbf{x}_{1}, \cdots, \mathbf{x}_{2 n}\right) \\
&=-2 \sum_{\substack{j, k=1 \\
j<k}}^{2 n} B\left(\left|\mathbf{x}_{j}-\mathbf{x}_{k}\right|\right) F_{2 n-2}\left(\mathbf{x}_{1}, \cdots, \mathbf{x}_{2 n}\right) . \\
& j \hat{k}
\end{aligned}
$$

Evaluation of the structures functions $S_{2 p}(r)=\left\langle(\theta(\mathrm{x}+\mathrm{r}, t)-\theta(\mathrm{x}, t))^{2 p}\right\rangle$ can in principle be carried out once the solution of (102) is known. However, solving these equations is a very difficult task and so far only perturbative methods have been successful in some regimes.

A simple dimensional argument suggests that a Kolmogorov-like theory would predict normal scaling exponents $\alpha_{p}=\frac{2-\xi}{2} p$. We will see below that this is not true for $p>2$.

Our task is to analyze the behavior of $F_{2 n}$ for small $\left|\mathbf{x}_{j}-\mathbf{x}_{k}\right|$. We will restrict ourselves to translation invariant solutions. 
¿From (102), $F_{2 n}$ has contributions from the inhomogeneous part $F_{2 n-2}$, and the homogeneous part, which at small distances are solutions of

$$
\mathcal{M}_{2 n}^{\mathcal{S C}} \tilde{F}_{2 n}=\sum_{j, k=1}^{2 n} d_{\alpha \beta}\left(\mathbf{x}_{j}-\mathbf{x}_{k}\right) \nabla_{\alpha}^{j} \nabla_{\beta}^{k} \tilde{F}_{2 n}=0
$$

where

$$
d_{\alpha \beta}(\mathbf{x})=D\left((d+\xi-1) \delta_{\alpha \beta}-\xi \frac{x_{\alpha} x_{\beta}}{r^{2}}\right) r^{\xi}
$$

(103) is obtained as the scaling limit of the homogeneous equation associated with (102). The solutions of (103) are called zero modes $[\mathbf{8}, \mathbf{1 9}, \mathbf{5 0}]$. This is an important concept that characterizes the origin of the anomalous scaling, and the leading order singularities of the correlation functions.

As an example, let us study the behavior of the 2-point function at $d=3 . \quad F_{2}$ satisfies

$$
-\frac{2}{r^{2}} \frac{\partial}{\partial r}\left(D r^{2+\xi} \frac{\partial F_{2}}{\partial r}\right)=B(r)
$$

It is easy to obtain from this equation that

$$
F_{2}(r)=C_{0}-C_{1} r^{2-\xi}+\cdots
$$

where $C_{0}, C_{1}$ are constants depending on $B$ and the neglected terms are of higher order. Hence we have for $S_{2}$

$$
S_{2}(r)=C_{2} r^{2-\xi}+\cdots
$$

This implies that $S_{2}$ obeys normal scaling. Note that the constant $C_{2}$ is in general non-universal.

Let us now look at the 4-point function $F_{4}\left(\mathbf{x}_{1}, \mathbf{x}_{2}, \mathbf{x}_{3}, \mathbf{x}_{4}\right)$ and let $\mathbf{x}_{j k}=\mathbf{x}_{j}-$ $\mathbf{x}_{k}, j, k=1, \cdots 4 . F_{4}$ has contributions from the "Gaussian" part:

$$
F_{2}\left(\mathbf{x}_{12}\right) F_{2}\left(\mathbf{x}_{34}\right)+F_{2}\left(\mathbf{x}_{13}\right) F_{2}\left(\mathbf{x}_{24}\right)+F_{2}\left(\mathbf{x}_{14}\right) F_{2}\left(\mathbf{x}_{23}\right),
$$

which gives rise to normal scaling, as well as the contribution from the zero modes which may be the dominant contribution to $S_{4}$. To find the precise form of the zero modes is a very difficult task. Both [50] and [19] resorted to perturbation techniques in either $\xi$ or $1 / d$. Here we follow [50] and write

$$
\tilde{F}=E_{0}+\xi G_{0}+O\left(\xi^{2}\right)
$$

where $E_{0}$ is the zero mode for the case when $\xi=0$. Using the notations $\nabla_{12}=\nabla_{\mathbf{x}_{12}}$, etc, we can write down an equation for $E_{0}$ :

$$
-\left(\Delta_{12}+\Delta_{23}+\Delta_{34}-\nabla_{12} \cdot \nabla_{23}-\nabla_{23} \cdot \nabla_{34}\right) E_{0}=0 .
$$

Substituting (110) into (103), we obtain an equation for $G_{0}$ :

$$
-\Delta_{4} G_{0}+\mathcal{L} E_{0}=0
$$

where

$$
\Delta_{4}=\Delta_{\mathbf{x}_{1}}+\cdots+\Delta_{\mathbf{x}_{4}}, \mathcal{L}=\sum_{j \neq k}\left(\delta_{\alpha \beta}-\frac{1}{2} \frac{x_{j k}^{\alpha} x_{j k}^{\beta}}{\left|\mathbf{x}_{j k}\right|^{2}}\right) \nabla_{j}^{\alpha} \nabla_{k}^{\beta}-\frac{1}{2} \Delta_{4}
$$


The precise form of $G_{0}$ is quite complicated. We refer to $[\mathbf{8}, \mathbf{5 0}]$ for the details of this calculation from which one obtains that $\tilde{F}$ must be homogeneous of degree

$$
\alpha_{4}=4-\frac{14}{5} \xi+O\left(\xi^{2}\right)=2 \alpha_{2}-\rho_{2},
$$

where $\rho_{2}=\frac{4}{5} \xi+O\left(\xi^{2}\right)$ is the anomalous exponent. Numerical results of Frisch et.al [49] has found very good agreement with (111).

\section{Stochastic Navier-Stokes Equation}

We are interested in two mathematical issues:

1. The existence and uniqueness of an invariant measure under physical conditions.

2. The limiting behavior of the invariant measure as viscosity approaches zero. Presently progress has only been made on the first issue. Here the main question is the condition on the stochastic forcing under which uniqueness of the invariant measure and consequently ergodicity of the dynamics can be proved.

4.1. Ergodicity When All Determining Modes are Forced. Consider

$$
\left\{\begin{array}{l}
\mathbf{u}_{t}+(\mathbf{u} \cdot \nabla) \mathbf{u}+\nabla p=\nu \Delta \mathbf{u}+\frac{\partial \mathbf{W}}{\partial t} \\
\nabla \cdot \mathbf{u}=0
\end{array}\right.
$$

We will restrict ourselves to two dimension, and we will take $\mathbf{W}$ to be of the form

$$
\mathbf{W}(\mathbf{x}, t)=\sum_{k} \sigma_{k} \beta_{k}(t, \omega) \mathbf{e}_{k}(\mathbf{x})
$$

where the $\beta_{k}$ 's are standard i.i.d complex-valued Brownian motion, and $\sigma_{k} \in \mathbb{C}$, are the amplitudes of the forcing, $\left\{\mathbf{e}_{k}(\mathbf{x})=\left(\begin{array}{c}-i k_{2} \\ i k_{1}\end{array}\right) \frac{e^{i k \cdot x}}{|k|}, k \in \mathbb{Z}\right\}$ are the complex eigen basis of $L^{2}$ divergence-free vector fields on $\mathbb{T}^{2}$, the two dimensional torus.

Define $B(\mathbf{u}, \mathbf{w})=-P_{\text {div }}(\mathbf{u} \cdot \nabla) \mathbf{w}, \quad \Lambda^{2} \mathbf{u}=-P_{\text {div }} \Delta \mathbf{u}$ where $P_{\text {div }}$ is the $L^{2}$ projection operator onto the space of divergence-free vector fields. Let $\mathcal{E}_{0}=\sum_{k}\left|\sigma_{k}\right|^{2}$, $\mathcal{E}_{1}=\left.\sum_{k}|k|^{2} \sigma_{k}\right|^{2}$. Writing $\mathbf{u}(\mathbf{x})=\sum_{k} u_{k} \mathbf{e}_{k}(\mathbf{x})$, we will define $\mathbb{H}^{\alpha}=\left\{\mathbf{u}=\left(\mathbf{u}_{k}\right)_{k \in \mathbb{Z}^{2}}\right.$, $\left.\sum_{k}|k|^{2 \alpha}\left|\mathbf{u}_{k}\right|^{2}<\infty\right\}$ and $\mathbb{L}^{2}=\mathbb{H}^{0}$.

We will use the standard probabilistic setup, namely we will work on a probability space $\left(\Omega, \mathcal{F}, \mathcal{F}_{t}, \mathbb{P}, \theta_{t}\right)$, where $(\Omega, \mathcal{F}, \mathbb{P})$ is the canonical probability space generated by all $\beta_{k}, \mathcal{F}_{t}$ is the $\sigma$-algebra generated by $\beta_{k}(s)$ for $s \leq t$, and $\theta_{t}$ is the shift operator on $\Omega$ defined by $\left(\theta_{t} d \beta_{k}\right)(s)=d \beta_{k}(s+t)$. Expectations with respect to $\mathbb{P}$ will be denoted by $\mathbb{E}$. We formally write (113) as an Ito differential equation in $\mathbb{L}^{2}$ :

$$
d \mathbf{u}(\mathbf{x}, t)+\nu \Lambda^{2} \mathbf{u}(\mathbf{x}, t) d t=B(\mathbf{u}, \mathbf{u}) d t+d \mathbf{W}(\mathbf{x}, t)
$$

Even though technically unnecessary sometimes, we will assume that $\sigma_{k}$ decays exponentially fast in $k$. This is reasonable from a physical viewpoint. Under this condition, it can be easily shown that for almost all realizations of the stochastic forcing, the dynamics (112) is well-defined. Furthermore, for $t \geq s$, let $\mathbf{u}\left(t, \omega ; s, \mathbf{u}_{0}\right)$ be the solution of (112) at time $t$ satisfying the initial condition $\mathbf{u}(s)=\mathbf{u}_{0}$, then (112) generates a continuous stochastic semi-flow on $\mathbb{L}^{2}$

$$
\varphi_{s, t}^{\omega}\left(\mathbf{u}_{0}\right)=\mathbf{u}\left(t, \omega ; s, \mathbf{u}_{0}\right)
$$

We will take the state space to be $\mathbb{L}^{2}$ equipped with the Borel $\sigma$-algebra. 
Definition. A measure $\mu(d u)$ on $\mathbb{L}^{2}$ is invariant for the stochastic flow (114) if for all bounded continuous functionals $F$ on $\mathbb{L}^{2}$ and $t>0$

$$
\int_{\mathbb{L}^{2}} F(\mathbf{u}) \mu(d u)=\int_{\mathbb{L}^{2}} \mathbb{E} F\left(\varphi_{0, t}^{\omega} \mathbf{u}\right) \mu(d u)
$$

Existence of an invariant measure is proved by Vishik and Fursikov [100], Flandoli and Maslowski [46]. The basic idea is to use the classical Krylov-Bogoliubov theorem asserting the existence of invariant measures for general dynamical systems on a compact state space. Here our state space is not compact, but since (112) is of parabolic type, the solution operator is compact and this turns out to be sufficient.

The first uniqueness result was also proved by Flandoli and Maslowski [46]. Their result requires that the forcing amplitude $\sigma_{k}$ satisfy

$$
C_{1}|k|^{-1 / 2} \leq\left|\sigma_{k}\right| \leq C_{2}|k|^{-3 / 8-\varepsilon}
$$

In this case the dynamics of the SNS (Stochastic Navier-Stokes) is dominated by noise. The nonlinear term can be viewed as a perturbation of the linear equation with noise. Such a condition is very rarely met in physical situations.

In his thesis, Mattingly proved that if the viscosity is sufficiently large, the one force, one solution principle holds and hence the invariant measure must be unique $[81,82]$.

In the more general case, E, Mattingly and Sinai proved

Theorem 13. [33] Assume that $\sigma_{k} \neq 0$ for $|k| \leq N$ where $N^{2} \geq \frac{\bar{C}^{2}}{2} \frac{\mathcal{E}_{0}}{\nu^{3}}$ and $\bar{C}$ is some absolute constant. Then (112) has a unique invariant measure on $\mathbb{L}^{2}$.

Results of a similar nature were also independently obtained by Kuksin and Shirikyan [65, 66], Bricmont, Kupiainen and Lefevere [17], see also [86].

Theorem 13 says that if all the "determining modes" are forced, then the invariant measure is unique and hence the dynamics is ergodic in the phase space.

The main idea of the proof is to show that for solutions that are defined over the time interval $(-\infty, \infty)$, their high modes can be uniquely represented by the past history of the low modes. This means that the dynamics of the Navier-Stokes equation on its infinite-dimensional phase space can be represented by a finitedimensional system, but with memory. Ergodicity is then established by proving that time averages of a continuous functional along a trajectory is independent of the initial data of the trajectory. This last step is done using the Girsanov formula.

Below we explain these two steps in more detail.

Step 1. Reduction to Gibbsian dynamics.

We show that the Markovian dynamics of the infinite-dimensional NavierStokes equations can be reduced to the Gibbsian dynamics of a finite-dimensional system. To this end, we define two subspaces.

$$
\mathbb{L}_{\ell}^{2}=\operatorname{span}\left\{\mathbf{e}_{k},|k| \leq N\right\}, \quad \mathbb{L}_{h}^{2}=\operatorname{span}\left\{\mathbf{e}_{k},|k|>N\right\}
$$

We will call $\mathbb{L}_{\ell}^{2}$ the set of low modes and $\mathbb{L}_{h}^{2}$ the set of high modes. Obviously $\mathbb{L}^{2}=\mathbb{L}_{\ell}^{2} \oplus \mathbb{L}_{h}^{2}$. Denote by $P_{\ell}$ and $P_{h}$ the projections onto the low and high mode spaces.

We write $\mathbf{u}(t)=(\mathbf{l}(t), \mathbf{h}(t)), P_{\ell} d \mathbf{W}(t)=d \mathbf{W}_{\ell}(t), P_{h} d \mathbf{W}(t)=d \mathbf{W}_{h}(t)$

$$
\begin{aligned}
d l(t) & =\left[-\nu \Lambda^{2} \mathbf{l}+P_{\ell} B(\mathbf{l}, \mathbf{l})\right] d t \\
& +\left[P_{\ell} B(\mathbf{l}, \mathbf{h})+P_{\ell} B(\mathbf{h}, \mathbb{l})+P_{\ell} B(\mathbf{h}, \mathbf{h})\right] d t+d \mathbf{W}_{\ell}(t)
\end{aligned}
$$




$$
\begin{aligned}
d \mathbf{h}(t) & =\left[-\nu \Lambda^{2} \mathbf{h}+P_{h} B(\mathbf{h}, \mathbf{h})\right] d t \\
& +\left[P_{h} B(\mathbf{l}, \mathbf{h})+P_{h} B(\mathbf{h}, \mathbf{l})+P_{h} B(\mathbf{l}, \mathbf{l})\right] d t+d \mathbf{W}_{h}(t)
\end{aligned}
$$

Define the set of "nice pasts" $U \subset C\left((-\infty, 0], \mathbb{L}^{2}\right)$ to consist of all $\mathbf{v}:(-\infty, 0] \rightarrow \mathbb{L}^{2}$ such that:

1. $\mathbf{v}(t)$ is in $\mathbb{H}^{2}$ for all $t \leq 0$

2. The energy averages correctly. More precisely,

$$
\lim _{t \rightarrow-\infty} \frac{1}{|t|} \int_{t}^{0}|\Lambda \mathbf{v}(s)|_{\mathbb{L}^{2}}^{2} d s=\frac{\mathcal{E}_{0}}{2 \nu}
$$

3. The energy fluctuations are typical. More precisely, there exists a $T=T(\mathbf{v})$ such that

$$
|\mathbf{v}(t)|_{\mathbb{L}^{2}}^{2} \leq \mathcal{E}_{0}+\max (|t|, T)^{\frac{2}{3}}
$$

for $t \leq 0$. It can be shown [33] that $U$ contains almost all of the trajectories defined on the whole time interval.

Given a function $\mathbf{l}$, defined up to time $t$, let us denote by $\Phi_{s, t}\left(\mathbf{l}, \mathbf{h}_{0}\right)$ the solution to (119) at time $t$ with initial condition $\mathbf{h}_{0}$ at time $s$ and the "forcing" $\mathbf{l}$.

LEMMA 4. [33] There exists an absolute positive constant $\mathcal{C}$ such that if $N^{2}>$ $\mathcal{C} \frac{\mathcal{E}_{0}}{\nu^{3}}$ then the following holds:

If there exists two solutions $\mathbf{u}_{1}(t)=\left(\mathbf{l}(t), \mathbf{h}_{1}(t)\right), \mathbf{u}_{2}(t)=\left(\mathbf{l}(t), \mathbf{h}_{2}(t)\right)$ corresponding to some (possibly different) realizations of the forcing and such that $\mathbf{u}_{1}, \mathbf{u}_{2} \in U$, then $\mathbf{u}_{1}=\mathbf{u}_{2}$, i.e. $\mathbf{h}_{1}=\mathbf{h}_{2}$.

Furthermore given a solution $\mathbf{u}(t)=(\mathbf{l}(t), \mathbf{h}(t)) \in U$, any $\mathbf{h}_{0} \in \mathbb{L}_{h}^{2}$, and $t \leq 0$ the following limit exists

$$
\lim _{t_{0} \rightarrow-\infty} \Phi_{t_{0}, t}\left(\mathbf{l}, \mathbf{h}_{0}\right)=\mathbf{h}^{*}
$$

and $\mathbf{h}^{*}=\mathbf{h}(t)$.

Proof. Let $\rho(t)=\mathbf{h}_{1}(t)-\mathbf{h}_{2}(t)$. From (119) we have

$$
\begin{aligned}
\frac{d \rho}{d t} & =-\nu \Lambda^{2} \boldsymbol{\rho}+P_{h} B\left(\mathbf{h}_{1}, \mathbf{h}_{1}\right)-P_{h} B\left(\mathbf{h}_{2}, \mathbf{h}_{2}\right)+P_{h} B(\mathbf{l}, \boldsymbol{\rho})+P_{h} B(\boldsymbol{\rho}, \mathbf{l}) \\
& =-\nu \Lambda^{2} \boldsymbol{\rho}+P_{h} B\left(\mathbf{l}+\mathbf{h}_{1}, \boldsymbol{\rho}\right)+P_{h} B\left(\boldsymbol{\rho}, \mathbf{l}+\mathbf{h}_{2}\right) \\
& =-\nu \Lambda^{2} \boldsymbol{\rho}+P_{h} B\left(\mathbf{u}_{1}, \boldsymbol{\rho}\right)+P_{h} B\left(\boldsymbol{\rho}, \mathbf{u}_{2}\right) .
\end{aligned}
$$

Taking the inner product with $\rho$, using the fact that $\left\langle P_{h} B\left(\mathbf{u}_{1}, \rho\right), \rho\right\rangle_{\mathbb{L}^{2}}=0$, gives

$$
\frac{1}{2} \frac{d}{d t}|\rho|_{L^{2}}^{2}=-\nu|\Lambda \rho|_{L^{2}}^{2}+\left\langle P_{h} B\left(\rho, \mathbf{u}_{2}\right), \boldsymbol{\rho}\right\rangle_{\mathbb{L}^{2}} .
$$

Using the fact that $[21]$

$$
\begin{aligned}
\left|\left\langle P_{h} B\left(\boldsymbol{\rho}, \mathbf{u}_{2}\right), \rho\right\rangle_{\mathbb{L}^{2}}\right| & \leq \hat{C}|\Lambda \rho|_{\mathbb{L}^{2}}|\rho|_{\mathbb{L}^{2}}\left|\Lambda \mathbf{u}_{2}\right|_{\mathbb{L}^{2}} \\
& \leq \frac{\nu}{2}|\Lambda \rho|_{\mathbb{L}^{2}}^{2}+\frac{\hat{C}^{2}}{2 \nu}|\rho|_{\mathbb{L}^{2}}^{2}\left|\Lambda \mathbf{u}_{2}\right|_{\mathbb{L}^{2}}^{2}
\end{aligned}
$$

we get

$$
\frac{1}{2} \frac{d}{d t}|\rho|_{\mathbb{L}^{2}}^{2} \leq-\frac{\nu}{2}|\Lambda \rho|_{\mathbb{L}^{2}}^{2}+\frac{\hat{C}^{2}}{2 \nu}\left|\Lambda \mathbf{u}_{2}\right|_{\mathbb{L}^{2}}^{2}|\rho|_{\mathbb{L}^{2}}^{2}
$$


Since $\boldsymbol{\rho}$ only contains modes with $|k|>N$, Poincaré inequality implies

$$
\frac{d}{d t}|\rho|_{\mathbb{L}^{2}}^{2} \leq\left(-\nu N^{2}+\frac{\hat{C}^{2}}{\nu}\left|\Lambda \mathbf{u}_{2}\right|_{\mathbb{L}^{2}}^{2}\right)|\rho|_{\mathbb{L}^{2}}^{2}
$$

Therefore we have, for $t_{0}<t<0$,

$$
|\rho(t)|_{\mathbb{L}^{2}}^{2} \leq\left|\rho\left(t_{0}\right)\right|_{\mathbb{L}^{2}}^{2} \exp \left\{-\nu N^{2}\left(t-t_{0}\right)+\frac{\hat{C}^{2}}{\nu} \int_{t_{0}}^{t}\left|\Lambda \mathbf{u}_{2}(s)\right|_{\mathbb{L}^{2}}^{2} d s\right\} .
$$

¿From the second assumption on functions in $U$, we know that

$$
\lim \frac{1}{t} \int_{-t}^{0}\left|\Lambda \mathbf{u}_{2}(s)\right|_{\mathbb{L}^{2}}^{2} d s=\frac{\mathcal{E}_{0}}{2 \nu}
$$

Hence for $t_{0}<T_{1}$, where $T_{1}$ depends on $t$ and $\mathbf{u}_{2}$, we have

$$
-\nu N^{2}\left(t-t_{0}\right)+\frac{\hat{C}^{2}}{\nu} \int_{t_{0}}^{t}\left|\Lambda \mathbf{u}_{2}(s)\right|_{\mathbb{L}^{2}}^{2} d s \leq-\frac{\gamma}{2}\left(t-t_{0}\right)
$$

where $\gamma=\nu N^{2}-\frac{\hat{C}^{2} \varepsilon_{0}}{2 \nu^{2}}$. If we set $\mathcal{C}=\frac{\hat{C}^{2}}{2}$, then our assumption on $N$ implies $\gamma>0$.

Now using the last property of paths in $U$ we have for any $t_{0} \leq T_{2}$,

$$
\begin{aligned}
|\rho(t)|_{\mathbb{L}^{2}}^{2} & \leq\left|\rho\left(t_{0}\right)\right|_{\mathbb{L}^{2}}^{2} \exp \left\{-\frac{\gamma}{2}\left(t-t_{0}\right)\right\} \\
& \leq 2\left[\mathcal{E}_{0}+\left|t_{0}\right|^{\frac{2}{3}}\right] \exp \left\{-\frac{\gamma}{2}\left(t-t_{0}\right)\right\} \rightarrow 0
\end{aligned}
$$

as $t_{0} \rightarrow-\infty$ where $T_{2}$ is some finite constant depending on $\mathbf{u}_{1}$ and $\mathbf{u}_{2}$. This completes the proof of the first part of the Lemma.

To prove the second part, observe that (121) only required control of $\int_{t_{0}}^{t}|\Lambda \mathbf{u}(s)|_{\mathbb{L}^{2}}^{2} d s$ for one of the two solutions. If we proceed as before letting the given solution $\mathbf{u}(t)$ play the role of $\mathbf{u}_{2}$ and the solution to (119) starting from $\mathbf{h}_{0}$ play the role of $\mathbf{u}_{1}$, then we obtain the estimate

$$
|\rho(t)|_{\mathbb{L}^{2}}^{2} \leq\left|\mathbf{h}\left(t_{0}\right)-\mathbf{h}_{0}\right|_{\mathbb{L}^{2}}^{2} \exp \left\{-\nu N^{2}\left(t-t_{0}\right)+\frac{\hat{C}^{2}}{\nu} \int_{t_{0}}^{t}|\Lambda \mathbf{u}(s)|_{\mathbb{L}^{2}}^{2} d s\right\} .
$$

Since $\mathbf{u}(t)=(\mathbf{l}(t), \mathbf{h}(t)) \in U$, the same reasoning as before shows that $\boldsymbol{\rho}(t)$ goes to zero as $t_{0} \rightarrow-\infty$. Hence the limit exists and equals $\mathbf{h}(t)$.

Denote by $\mathcal{P}$ the projection of $U$ to low modes. Given an "initial low mode past" $L^{0}$ in $\mathcal{P}$, we can then uniquely construct the high mode at $t=0$, denoted by $\mathbf{h}(0)=\Phi_{0}\left(L^{0}\right)$, and the solution to the full system (118), (119), denoted by $\mathbf{u}(t)=(\mathbf{l}(t), \mathbf{h}(t))$. We will denote the trajectory of the low mode on the time interval $(-\infty, t]$ by $L^{t}$ and $\mathbf{h}(t)=\Phi_{t}\left(L^{t}\right)$. Using these notations we can rewrite (118) and (119) as a history-dependent finite dimensional system

$$
d \mathbb{l}(t)=\left[-\nu \Lambda^{2} \mathbb{l}(t)+P_{\ell} B(\mathbb{l}(t), \mathbb{l}(t))+G\left(\mathbb{l}(t), \Phi_{t}\left(L^{t}\right)\right)\right] d t+d \mathbb{W}(t)
$$

where

$$
G(\mathbf{l}, \mathbf{h})=P_{\ell} B(\mathbf{l}, \mathbf{h})+P_{\ell} B(\mathbf{h}, \mathbf{l})+P_{\ell} B(\mathbf{h}, \mathbf{h}) .
$$

Thus we have a closed formulation of the dynamics on the low modes given an initial past in $L^{0} \in \mathcal{P}$. We write $L^{t}=\mathrm{S}_{t}^{\omega} L^{0}$. We reiterate that $L^{t}$ is the entire trajectory from time $t$ back to $-\infty$ whereas $1(t)$ is simply the value of the low modes at time $t$. 
(123) is not Markovian, but rather Gibbsian (see the discussions in $\S 4$ of [33]). Notice that the stochastic forcing in (123) is non-degenerate which implies ergodicity if it were Markovian.

Step 2. Ergodicity of the finite dimensional Gibbsian dynamics.

Our task is reduced to showing that the memory effect in (123) is not strong enough to destroy ergodicity.

In order to show this, we prove that the measures induced by different initial pasts for (123) are equivalent to each other.

The key idea is to realize that the Girsanov transformation can still be used for memory-dependent systems. The main technical point is to cut-off the nonlinear growth term so that the Novikov condition for the application of the Girsanov formula is satisfied. For details we refer to [33].

It is proved in $[\mathbf{8 3}]$ that the empirical measures, starting from arbitrary initial condition, converge to the unique invariant measure exponentially fast.

4.2. General Stochastic Dissipative PDEs. The philosophy presented in the last section is applicable to general stochastic dissipative PDEs such as the Ginzburg-Landau equation, the Kuramoto-Sivashinsky equation and the CahnHilliard equation. The general result, proved in [31], states that if all determining modes are forced, the invariant measure is unique and the dynamics is ergodic. Related results are also found in $[64,86,83]$.

4.3. Ergodicity with Minimum Stochastic Forcing. The condition in Theorem 13 is still unsatisfactory since the number of modes that need to be forced goes to infinity as $\nu \rightarrow 0$. The physically more interesting situation is when only the large scale modes are forced. It is then natural to ask the question: what is the smallest set of modes that have to be forced in order to have ergodicity?

To answer this question, let us consider the two-dimensional case and use the vorticity formulation of the Navier-Stokes equation $\left(\eta=\frac{\partial u_{1}}{\partial x_{2}}-\frac{\partial u_{2}}{\partial x_{1}}\right)$.

$$
d \eta=\{\nu \Delta \eta-(\mathbf{u} \cdot \nabla) \eta\} d t+d f
$$

We will consider the case when the stochastic forcing has the following form.

$$
d f=\sum_{\mathbf{k} \in K} \sigma_{\mathbf{k}} \cos (\mathbf{k} \cdot \mathbf{x}) d B_{\mathbf{k}}(t)+\sum_{\mathbf{k} \in K} \gamma_{\mathbf{k}} \sin (\mathbf{k} \cdot \mathbf{x}) d \beta_{\mathbf{k}}(t)
$$

where the $B_{\mathbf{k}}$ 's and $\beta_{\mathbf{k}}$ 's are independent standard Wiener processes defined on a probability space $\{\Omega, F, \mathbb{P}\}$. Here $\mu_{\mathbf{k}}$ and $\gamma_{\mathbf{k}}$ are positive constants representing the amplitude of the forcing. $K$ is the set of modes that are forced. We will be primarily interested in the case when $K$ contains very few low modes.

Writing $\eta(\mathbf{x}, t)=\sum_{\mathbf{k}} \eta_{\mathbf{k}}(t) \cos (\mathbf{k} \cdot \mathbf{x})+\sum_{\mathbf{k}} \sigma_{\mathbf{k}}(t) \sin (\mathbf{k} \cdot \mathbf{x})$ where the index $\mathbf{k}$ is in the first quadrant. We can rewrite (125) as

$$
\begin{aligned}
d \eta_{\mathbf{l}} & =\left\{-\nu|1|^{2} \eta_{\mathbf{l}}+\sum_{\mathbf{j}+\mathbf{k}=\mathbf{l}} \frac{\mathbf{j}^{\perp} \cdot \mathbf{k}}{|\mathbf{j}|^{2}}\left(-\eta_{\mathbf{j}} \eta_{\mathbf{k}}+\mu_{\mathbf{j}} \mu_{\mathbf{k}}\right)\right. \\
& \left.+\sum_{\mathbf{j}-\mathbf{k}=\mathbf{l}} \frac{\mathbf{j}^{\perp} \cdot \mathbf{k}}{|\mathbf{j}|^{2}}\left(\eta_{\mathbf{j}} \eta_{\mathbf{k}}+\mu_{\mathbf{j}} \mu_{\mathbf{k}}\right)\right\} d t+\sigma_{\mathbf{l}} d B_{\mathbf{l}}
\end{aligned}
$$




$$
\begin{aligned}
d \mu_{\mathbf{l}} & =\left\{-\nu \mid \mathbb{1}^{2} \mu_{\mathbf{l}}-\sum_{\mathbf{j}+\mathbf{k}=\mathbf{l}} \frac{\mathbf{j}^{\perp} \cdot \mathbf{k}}{|\mathbf{j}|^{2}}\left(\eta_{\mathbf{j}} \mu_{\mathbf{k}}+\mu_{\mathbf{j}} \eta_{\mathbf{k}}\right)\right. \\
& \left.+\sum_{\mathbf{j}-\mathbf{k}=\mathbf{l}} \frac{\mathbf{j}^{\perp} \cdot \mathbf{k}}{|\mathbf{j}|^{2}}\left(-\eta_{\mathbf{j}} \mu_{\mathbf{k}}+\mu_{\mathbf{j}} \eta_{\mathbf{k}}\right)\right\} d t+\gamma_{\mathbf{l}} d \eta_{\mathbf{l}}
\end{aligned}
$$

where $\mathbf{j}^{\perp}=\left(-j_{2}, j_{1}\right)$. Here and in the rest of this section, we adopt the convention that the summation is done only over terms in which the indices $\mathbf{j}, \mathbf{k}, \mathbf{l}$ are in the first quadrant.

E and Mattingly [32] considered finite dimensional approximations of (126) and (127):

$$
\begin{aligned}
d \eta_{\mathbf{l}} & =\left\{-\nu|\mathbf{1}|^{2} \eta_{\mathbf{l}}+\sum_{\mathbf{j}+\mathbf{k}=\mathbf{l}}^{N} \frac{\mathbf{j}^{\perp} \cdot \mathbf{k}}{|\mathbf{j}|^{2}}\left(-\eta_{\mathbf{j}} \eta_{\mathbf{k}}+\mu_{\mathbf{j}} \mu_{\mathbf{k}}\right)\right. \\
& \left.+\sum_{\mathbf{j}-\mathbf{k}=\mathbf{l}}^{N} \frac{\mathbf{j}^{\perp} \cdot \mathbf{k}}{|\mathbf{j}|^{2}}\left(\eta_{\mathbf{j}} \eta_{\mathbf{k}}+\mu_{\mathbf{j}} \mu_{\mathbf{k}}\right)\right\} d t+\sigma_{\mathbf{l}} d B_{\mathbf{l}} \\
d \mu_{\mathbf{l}} & =\left\{-\nu|\mathbf{1}|^{2} \mu_{\mathbf{l}}-\sum_{\mathbf{j}+\mathbf{k}=\mathbf{l}}^{N} \frac{\mathbf{j}^{\perp} \cdot \mathbf{k}}{|\mathbf{j}|^{2}}\left(\eta_{\mathbf{j}} \mu_{\mathbf{k}}+\mu_{\mathbf{j}} \eta_{\mathbf{k}}\right)\right. \\
& \left.+\sum_{\mathbf{j}-\mathbf{k}=\mathbf{l}}^{N} \frac{\mathbf{j}^{\perp} \cdot \mathbf{k}}{|\mathbf{j}|^{2}}\left(-\eta_{\mathbf{j}} \mu_{\mathbf{k}}+\mu_{\mathbf{j}} \eta_{\mathbf{k}}\right)\right\} d t+\gamma_{\mathbf{l}} d \eta_{\mathbf{l}}
\end{aligned}
$$

where $\sum^{N}$ means that the sum is over indices $\mathbf{j}, \mathbf{k}, \mathbf{1}$ such that $|\mathbf{j}|_{\infty},|\mathbf{k}|_{\infty},|\mathbf{1}|_{\infty} \leq N$.

The main result of [32] is the following.

Theorem 14. Let $K_{1}=\{(0,1),(1,1)\}, K_{2}=\{(1,0),(1,1)\}$. If $K \supset K_{1}$, or $K \supset K_{2}$ then (128) and (129) has a unique invariant measure.

At the present time, there are still difficulties in extending Theorem 14 to the full Navier-Stokes equation. The difficulty seems to be technical, in adapting Malliavin calculus to the PDE. Some progress has been made in this direction in [39].

It is not clear whether the condition in Theorem 14 is sharp. There are two cases that are not covered by Theorem 14 . One is when $K=\{(1,0)\}$. The other is when $K=\{(1,0),(0,1)\}$. It would be interesting to understand what happens in these two cases.

Again the existence of an invariant measure follows from standard compactness results. The proof of uniqueness uses the following result of Harris [53].

Let $\left\{x_{n}, n=0,1, \ldots\right\}$ be a Markov process on a topological space $\mathbb{X}$ with Borel $\sigma$-algebra $\mathfrak{B}$. The Markov process $\left\{x_{n}, n=0,1, \ldots\right\}$ is said to satisfy Harris' condition if there exists a $\sigma$-finite measure $m$ on $\mathbb{X}$ such that if $m(E)>0, E \in \mathfrak{B}$, then

$$
\mathbb{P}_{x_{0}}\left\{x_{n} \in E \text { infinitely often }\right\}=1
$$


for all starting points $x_{0}$ in $\mathbb{X}$. Under this condition Harris proved that there is a measure $Q$, unique up to a constant multiplier, which solves the equation

$$
Q(E)=\int_{\mathbb{X}} P(x, E) Q(d x), \text { for } E \in \mathfrak{B}
$$

where $P(x, \cdot)$ is the transition probability distribution of the Markov process.

To establish Harris' condition, we prove the following:

1. Starting from any initial position, the dynamics enters any neighborhood of the origin infinitely often.

2. The transition probability distribution has a smooth density.

The main idea for proving (1) is to observe that in the absence of forcing, solutions of (128)-(129) decay exponentially fast due to the viscous term. This decay still holds when the forcing is small. The main idea for proving (2) is to prove that the Fokker-Planck operator associated with (128)-(129) is hypoelliptic.

Lemma 5. Assume that $K_{1} \subset K$ or $K_{2} \subset K$. Then the Markov process (128)(129) has a transition density $p_{t}(x, y)$ which is $C^{\infty}$ in $(x, y, t)$ for $t>0$.

LeMma 6. Fix a $\Delta t>0$ and an open neighborhood $U_{0}$ of the origin. Then given any initial condition $\eta_{0} \in \mathbb{R}^{(N+1)^{2}-1}$,

$$
\mathbb{P}_{\eta_{0}}\left\{\eta(n \Delta t) \in U_{0} \text { for infinitely many } n\right\}=1 \text {. }
$$

Using these lemmas uniqueness of the invariant measure follows quickly (see [32]).

We will not give the detailed proofs of these results here. Instead we will reproduce the calculation that leads to the hypoellipticity of the Fokker-Planck operator.

(128)-(129) is a degenerate diffusion process with analytic coefficients. The regularity of the transition density of such processes can be studied using Malliavin calculus. If we write the Fokker-Planck operator (the generator) of a diffusion process in the form

$$
L=X_{0}+\frac{1}{2} \sum_{j=1}^{l} X_{j}^{2}
$$

then the transition density is smooth if at each point of the state space if

$$
\begin{aligned}
& X_{j}, j=1,2 \cdots l \\
& {\left[X_{j}, X_{k}\right], j, k=0,1, \cdots l} \\
& {\left[X_{j},\left[X_{k_{1}}, X_{k_{2}}\right]\right], j, k_{1}, k_{2}=0,1, \cdots l}
\end{aligned}
$$

span the tangent space $\left[\mathbf{7 0}, \mathbf{5 4}, \mathbf{6 7}, \mathbf{8 7}\right.$. Notice that we allow $X_{0}$ to enter the higher-order brackets. This condition can be restated as: the ideal generated by $X_{1}, X_{2} \cdots X_{l}$ in the Lie algebra generated $X_{0}, X_{1}, X_{2}, \cdots X_{l}$ must have full rank at every point. We denote by $\operatorname{SPAN}(\eta)$ the ideal generated by $X_{1}, X_{2}, \cdots X_{l}$ at the point $\eta$. 
The Fokker-Planck operator for (128)--(129) can be written as

$$
L=X_{0}+\frac{1}{2} \sum_{\mathbf{k} \in K}^{N} \sigma_{\mathbf{k}}^{2}\left(\frac{\partial}{\partial \alpha_{\mathbf{k}}}\right)^{2}+\frac{1}{2} \sum_{\mathbf{k} \in K}^{N} \gamma_{\mathbf{k}}^{2}\left(\frac{\partial}{\partial \beta_{\mathbf{k}}}\right)^{2}
$$

where

$$
\begin{aligned}
X_{0} & =\sum_{\mathbf{l}}^{N}\left\{\sum_{\mathbf{j}+\mathbf{k}=\mathbf{l}}^{N} \frac{\mathbf{j}^{\perp} \cdot \mathbf{k}}{|\mathbf{j}|^{2}}\left(-\alpha_{\mathbf{j}} \alpha_{\mathbf{k}}+\beta_{\mathbf{j}} \alpha_{\mathbf{k}}\right)+\sum_{\mathbf{j}=\mathbf{k}=\mathbf{l}}^{N} \frac{\mathbf{j}^{\perp} \cdot \mathbf{k}}{|\mathbf{j}|^{2}}\left(\alpha_{\mathbf{j}} \alpha_{\mathbf{k}}+\beta_{\mathbf{j}} \beta_{\mathbf{k}}\right)-\nu|\mathbf{1}|^{2} \alpha_{\mathbf{l}}\right\} \frac{\partial}{\partial \alpha_{\mathbf{l}}} \\
& +\sum_{\mathbf{l}}^{N}\left\{\sum_{\mathbf{j}+\mathbf{k}=\mathbf{l}}^{N} \frac{\mathbf{j}^{\perp} \cdot \mathbf{k}}{|\mathbf{j}|^{2}}\left(-\alpha_{\mathbf{j}} \beta_{\mathbf{k}}-\beta_{\mathbf{j}} \alpha_{\mathbf{k}}\right)+\sum_{\mathbf{j}-\mathbf{k}=\mathbf{l}}^{N} \frac{\mathbf{j}^{\perp} \cdot \mathbf{k}}{|\mathbf{j}|^{2}}\left(-\alpha_{\mathbf{j}} \beta_{\mathbf{k}}+\beta_{\mathbf{j}} \alpha_{\mathbf{k}}\right)-\nu|1|^{2} \beta_{\mathbf{l}}\right\} \frac{\partial}{\partial \beta_{\mathbf{l}}}
\end{aligned}
$$

We can now calculate

$$
\begin{aligned}
& X_{\mathbf{m}}=\left[X_{0}, \frac{\partial}{\partial \alpha_{\mathbf{m}}}\right] \\
& =\sum_{\mathbf{l}}^{N}\left\{\alpha_{\mathbf{l}-\mathbf{m}}\left(\frac{1}{|\mathbf{m}|^{2}}-\frac{1}{|\mathbf{1 - \mathbf { m }}|^{2}}\right)+\alpha_{\mathbf{m}-\mathbf{1}} \frac{1}{|\mathbf{m}|^{2}}+\alpha_{\mathbf{l}+\mathbf{m}} \frac{1}{|\mathbf{l}+\mathbf{m}|^{2}}\right\}\left(\mathbf{1}^{\perp} \cdot \mathbf{m}\right) \frac{\partial}{\partial \alpha_{\mathbf{l}}} \\
& +\sum_{\mathbf{l}}^{N}\left\{\beta_{\mathbf{l}-\mathbf{m}}\left(\frac{1}{|\mathbf{m}|^{2}}-\frac{1}{|\mathbf{l}-\mathbf{m}|^{2}}\right)-\beta_{\mathbf{m}-\mathbf{1}} \frac{1}{|\mathbf{m}|^{2}}+\beta_{\mathbf{l}+\mathbf{m}} \frac{1}{|\mathbf{I}+\mathbf{m}|^{2}}\right\}\left(\mathbf{l}^{\perp} \cdot \mathbf{m}\right) \frac{\partial}{\partial \beta_{\mathbf{l}}} \\
& -\nu|\mathbf{m}|^{2} \frac{\partial}{\partial \alpha_{\mathbf{m}}} \\
& Y_{\mathbf{m}}=\left[X_{0}, \frac{\partial}{\partial \beta_{\mathbf{m}}}\right] \\
& =\sum_{\mathbf{l}}^{N}\left\{\beta_{\mathbf{l}-\mathbf{m}}\left(-\frac{1}{|\mathbf{m}|^{2}}+\frac{1}{|\mathbf{1}-\mathbf{m}|^{2}}\right)+\beta_{\mathbf{m}-\mathbf{1}} \frac{1}{|\mathbf{m}|^{2}}+\beta_{\mathbf{m}+\mathbf{1}} \frac{1}{|\mathbf{l}+\mathbf{m}|^{2}}\right\}\left(\mathbf{l}^{\perp} \cdot \mathbf{m}\right) \frac{\partial}{\partial \alpha_{\mathbf{l}}} \\
& +\sum_{\mathbf{l}}^{N}\left\{\alpha_{\mathbf{l}-\mathbf{m}}\left(\frac{1}{|\mathbf{m}|^{2}}-\frac{1}{|\mathbf{l}-\mathbf{m}|^{2}}\right)+\alpha_{\mathbf{m}-\mathbf{1}} \frac{1}{|\mathbf{m}|^{2}}-\alpha_{\mathbf{m}+\mathbf{l}} \frac{1}{|\mathbf{l}+\mathbf{m}|^{2}}\right\}\left(\mathbf{l}^{\perp} \cdot \mathbf{m}\right) \frac{\partial}{\partial \beta_{\mathbf{l}}} \\
& -\nu|\mathbf{m}|^{2} \frac{\partial}{\partial \beta_{\mathbf{m}}}
\end{aligned}
$$

We emphasize our convention that the terms are present in the sums only if the indices are in the first quadrant. If we denote by $Q_{N}=\left\{\mathbf{j}=\left(j_{1}, j_{2}\right), 0 \leq j_{1}, j_{2} \leq\right.$ $\left.N,\left(j_{1}, j_{2}\right) \neq(0,0)\right\}$, then the first sum in $X_{\mathbf{m}}$ should be written as

$$
\begin{aligned}
& \sum_{\mathbf{l}, \mathbf{l}-\mathbf{m} \in Q_{N}} \alpha_{\mathbf{l}-\mathbf{m}}\left(\frac{1}{|\mathbf{m}|^{2}}-\frac{1}{|\mathbf{l}-\mathbf{m}|^{2}}\right)\left(\mathbf{l}^{\perp} \cdot \mathbf{m}\right) \frac{\partial}{\partial \alpha_{\mathbf{l}}}+ \\
+ & \sum_{\mathbf{l}, \mathbf{m}-\mathbf{l} \in Q_{N}} \alpha_{\mathbf{m}-\mathbf{l}} \frac{1}{|\mathbf{m}|^{2}}\left(\mathbf{l}^{\perp} \cdot \mathbf{m}\right) \frac{\partial}{\partial \alpha_{\mathbf{l}}}+ \\
+ & \sum_{\mathbf{l}, \mathbf{l}+\mathbf{m} \in Q_{N}} \alpha_{\mathbf{l}+\mathbf{m}} \frac{1}{|\mathbf{1}+\mathbf{m}|^{2}}\left(\mathbf{l}^{\perp} \cdot \mathbf{m}\right) \frac{\partial}{\partial \alpha_{\mathbf{l}}},
\end{aligned}
$$

and similarly for the other terms. 
We then have

$$
\begin{aligned}
& {\left[X_{\mathbf{m}}, \frac{\partial}{\partial \beta_{\mathbf{k}}}\right]=\left(\mathbf{k}^{\perp} \cdot \mathbf{m}\right)\left\{\left(\frac{1}{|\mathbf{m}|^{2}}-\frac{1}{|\mathbf{k}|^{2}}\right) \frac{\partial}{\partial \beta_{\mathbf{m}+\mathbf{k}}}+\frac{1}{|\mathbf{m}|^{2}} \frac{\partial}{\partial \beta_{\mathbf{m}-\mathbf{k}}}+\frac{1}{|\mathbf{k}|^{2}} \frac{\partial}{\partial \beta_{\mathbf{k}-\mathbf{m}}}\right\}} \\
& {\left[X_{\mathbf{m}}, \frac{\partial}{\partial \alpha_{\mathbf{k}}}\right]=\left(\mathbf{k}^{\perp} \cdot \mathbf{m}\right)\left\{\left(\frac{1}{|\mathbf{m}|^{2}}-\frac{1}{|\mathbf{k}|^{2}}\right) \frac{\partial}{\partial \alpha_{\mathbf{m}+\mathbf{k}}}-\frac{1}{|\mathbf{m}|^{2}} \frac{\partial}{\partial \alpha_{\mathbf{m}-\mathbf{k}}}+\frac{1}{|\mathbf{k}|^{2}} \frac{\partial}{\partial \alpha_{\mathbf{k}-\mathbf{m}}}\right\}} \\
& {\left[Y_{\mathbf{m}}, \frac{\partial}{\partial \beta_{\mathbf{k}}}\right]=\left(\mathbf{k}^{\perp} \cdot \mathbf{m}\right)\left\{-\left(\frac{1}{|\mathbf{m}|^{2}}-\frac{1}{|\mathbf{k}|^{2}}\right) \frac{\partial}{\partial \alpha_{\mathbf{m}+\mathbf{k}}}-\frac{1}{|\mathbf{m}|^{2}} \frac{\partial}{\partial \alpha_{\mathbf{m}-\mathbf{k}}}+\frac{1}{|\mathbf{k}|^{2}} \frac{\partial}{\partial \alpha_{\mathbf{k}-\mathbf{m}}}\right\}} \\
& {\left[Y_{\mathbf{m}}, \frac{\partial}{\partial \alpha_{\mathbf{k}}}\right]=\left(\mathbf{k}^{\perp} \cdot \mathbf{m}\right)\left\{\left(\frac{1}{|\mathbf{m}|^{2}}-\frac{1}{|\mathbf{k}|^{2}}\right) \frac{\partial}{\partial \beta_{\mathbf{m}+\mathbf{k}}}-\frac{1}{|\mathbf{m}|^{2}} \frac{\partial}{\partial \beta_{\mathbf{m}-\mathbf{k}}}-\frac{1}{|\mathbf{k}|^{2}} \frac{\partial}{\partial \beta_{\mathbf{k}-\mathbf{m}}}\right\}}
\end{aligned}
$$

Again the same summation convention as we discussed above applies.

Now fix any $\eta \in \mathbb{R}^{(N+1)^{2}-1}$. Take $\mathbf{m}=(1,1), \mathbf{k}=(0,1)$, then we have

$$
\begin{aligned}
& {\left[X_{\mathbf{m}}, \frac{\partial}{\partial \alpha_{\mathbf{k}}}\right]=\frac{1}{2}\left\{\frac{\partial}{\partial \alpha_{(1,2)}}+\frac{\partial}{\partial \alpha_{(1,0)}}\right\}} \\
& {\left[Y_{\mathbf{m}}, \frac{\partial}{\partial \beta_{\mathbf{k}}}\right]=-\frac{1}{2}\left\{\frac{\partial}{\partial \alpha_{(1,2)}}-\frac{\partial}{\partial \alpha_{(1,0)}}\right\}}
\end{aligned}
$$

Therefore if $K_{1} \subset K$, then $\frac{\partial}{\partial \alpha_{(1,2)}}, \frac{\partial}{\partial \alpha_{(1,0)}} \in \operatorname{SPAN}(\eta)$. Similarly take $\mathbf{k}=(1,0), \mathbf{m}=$ $(1,2)$, we find $\frac{\partial}{\partial \alpha_{(0,2)}} \in \operatorname{SPAN}(\eta)$. Proceeding in this way, we find $\frac{\partial}{\partial \alpha_{(1, l)}}, \frac{\partial}{\partial \alpha_{(0, l)}} \in$ $\operatorname{SPAN}(\eta)$, for $l \leq N$. Take $\mathbf{k}=(1,0), \mathbf{m}=(1, l)$, we get $\frac{\partial}{\partial \alpha_{(2, l)}} \in \operatorname{SPAN}(\eta)$, for $l>0$. Take $\mathbf{k}=(2,1)$ and $\mathbf{m}=(0,1)$ we get $\frac{\partial}{\partial \alpha_{(2,0)}} \in \operatorname{SPAN}(\eta)$. By induction, we obtain $\frac{\partial}{\partial \alpha_{(k, l)}}, \frac{\partial}{\partial \beta_{(k, l)}} \in \operatorname{SPAN}(\eta)$ for all $0 \leq k, l \leq N,(k, l) \neq(0,0)$.

This establishes hypoellipticity in the case when $K_{1} \subset K$. The case when $K_{2} \subset K$ can be dealt with in the same way.

\section{Regularity and Dissipation}

Formally, at the infinite Reynolds number limit, the Navier-Stokes equation reduce to Euler's equation which takes the form

$$
\left\{\begin{array}{l}
\mathbf{u}_{t}+(\mathbf{u} \cdot \nabla) \mathbf{u}+\nabla p=0 \\
\nabla \cdot \mathbf{u}=0
\end{array}\right.
$$

in the absence of forcing. For solutions of (2) and (133), one can define the energy $\frac{1}{2} \int_{D}|\mathbf{u}|^{2} d^{3} x$. For smooth solutions of (133), energy is conserved:

$$
\frac{1}{2} \int_{D}|\mathbf{u}(\mathbf{x}, t)|^{2} d^{3} x=\frac{1}{2} \int_{D}|\mathbf{u}(\mathbf{x}, 0)|^{2} d^{3} x
$$

if there is no boundary effects. To prove (134), multiply (133) by $\mathbf{u}$ and integrate by parts. However, turbulence theory is concerned with solutions of (133) that dissipates energy. In fact, one of the basic assumptions in turbulence theory is that under a fixed external forcing, $\bar{\varepsilon}=\left\langle\nu\left|\nabla \mathbf{u}^{\nu}\right|^{2}\right\rangle$ the energy dissipation rate stays finite in the limit $\nu \rightarrow 0$. Hence the limiting invariant measure, if exists, supports singular Euler flows for which $\left\langle|\nabla \mathbf{u}|^{2}\right\rangle=+\infty$ and energy is decreased in the absence of forcing. This was pointed out by Onsager [88] in 1949. This is the striking property shared by all three problems that we discussed: the hydrodynamic turbulence in three dimension, the Burgers turbulence and the turbulent transport of passive scalar fields, namely that formally in the inviscid limit, smooth solutions 
conserve energy, but our interest is on solutions that dissipate energy. Hence they cannot be smooth and we are interested in their singularities. In the case of the inviscid Burgers equation (the Burgers-Hopf equation)

$$
u_{t}+\frac{1}{2}\left(u^{2}\right)_{x}=0
$$

the energy is $\frac{1}{2} \int u^{2} d x$, which is conserved for smooth solutions, aside from boundary effects. However, it is well-known that solutions of (135) typically contain jump discontinuities called shocks. Physically relevant jump discontinuities have to be defined as the zero viscosity limit of the viscous problem

$$
u_{t}+\frac{1}{2}\left(u^{2}\right)_{x}=\nu u_{x x}
$$

as $\nu \rightarrow 0$. Such solutions satisfy the so-called entropy condition [71] which dissipates energy at the shocks. The statistics of the shocks give rise to the interesting behavior of the correlation functions.

Similarly, for the passive scalar transport equation in the "inviscid limit",

$$
\theta_{t}+(\mathbf{u} \cdot \nabla) \theta=0
$$

If $\mathbf{u}$ is incompressible $\nabla \cdot \mathbf{u}=0$ then the energy, defined as $\frac{1}{2} \int \theta^{2} d^{3} x$, is conserved when $\mathbf{u}$ is smooth. However, when $\mathbf{u}$ fails to be Lipschitz continuous, such as the case of the Kraichnan model, the generalized flow associated with (137) is in general non-degenerate and we have

$$
\begin{aligned}
\int \theta^{2}(\mathbf{x}, t) d x & =\iiint \theta_{0}(\mathbf{y}) \theta_{0}(\mathbf{z}) g(\mathbf{x}, t \mid \mathbf{y}, 0) g(\mathbf{x}, t \mid \mathbf{z}, 0) d y d z d x \\
& <\int \theta_{0}^{2}(\mathbf{x}) d x
\end{aligned}
$$

We see in Section 3 that $\theta$ is in general only Hölder continuous.

In [88], Onsager conjectured that the solutions of the 3D incompressible Euler's equation conserve energy if they are spatially Hölder continuous with exponent larger than $1 / 3$, and cease to conserve energy if the exponent is less than $1 / 3$. The first half of this statement was proved in [22] in its sharp form formulated in terms of Besov spaces. For simplicity of presentation, we will assume periodic boundary condition on the domain $D$.

THEOREM 15. [22]. Let $\mathbf{u}=\left(u_{1}, u_{2}, u_{3}\right) \in L^{3}\left([0, T], B_{3}^{s, \infty}(D)\right) \cap C\left([0, T], L^{2}(D)\right)$ be a weak solution of the $3 D$ incompressible Euler's equation, i.e.

$$
\begin{aligned}
& -\int_{0}^{T} \int_{D} u_{\alpha}(\mathbf{x}, t) \frac{\partial}{\partial t} \psi_{\alpha}(\mathbf{x}, t) d^{3} x d t-\int_{D} u_{\alpha}(\mathbf{x}, 0) \psi_{\alpha}(\mathbf{x}, 0) d^{3} x \\
& -\int_{0}^{T} \int_{D} u_{\alpha}(\mathbf{x}, t) u_{\beta}(\mathbf{x}, t) \nabla_{\alpha} \psi_{\beta}(\mathbf{x}, t) d^{3} x d t-\int_{0}^{T} \int_{D} p(\mathbf{x}, t) \nabla_{\alpha} \psi_{\alpha}(\mathbf{x}, t) d^{3} x d t=0,
\end{aligned}
$$

(summation convention is used) for every test function $\psi=\left(\psi_{1}, \psi_{2}, \psi_{3}\right) \in C^{\infty}(D \times$ $[0, T))$ with compact support. If $s>\frac{1}{3}$, then

$$
\int_{D}|\mathbf{u}(\mathbf{x}, t)|^{2} d^{3} x=\int_{D}|\mathbf{u}(\mathbf{x}, 0)|^{2} d^{3} x, \quad \text { for } t \in[0, T) .
$$

Besov space is the natural setting for formulating this result since its definition closely resembles the definition of structure functions except that the ensemble 
average is replaced by the spatial average. In fact, in more physical terms, Theorem 15 states that if

$$
\left(\int_{D}|u(\mathbf{x}+\mathbf{r}, t)-u(\mathbf{x}, t)|^{3} d^{3} x\right)^{1 / 3} \leq C|\mathbf{r}|^{\alpha},
$$

for $\alpha>1 / 3$, then $\bar{\varepsilon}=0$. This is the deterministic analog of Kolmogorov's 4/5 law. It is clear from this that Theorem 15 is sharp. Furthermore, Theorem (15) suggests that in the inviscid limit, turbulent velocity fields live in a space close to $B_{3}^{1 / 3, \infty}$.

This result further suggests that the typical "turbulent" velocity fields, the ones that Kolmogorov was interested in when he discussed the inertial range, are the smoothest velocity fields that dissipate energy. As far as regularity is concerned, they live at the boundary between conservative and dissipative weak solutions.

An exception to this scenario might be two dimensional turbulence. It is easy to prove, using the techniques in [25], that for the two-dimensional Euler's equation the enstrophy $\int \omega^{2} d x$ is conserved as long as it is finite. This observation was made by $\mathrm{E}$ and Vanden-Eijnden (see [41]). It is likely that there are no intrinsic dissipation mechanism present in the two dimensional Euler equation, and dissipation has to be added in order for the forced system to have an invariant measure.

\section{Conclusions}

Stochastic PDEs provide the natural framework for studying the longtime behavior of complex systems. In this regard, we have only touched upon one type of behavior: the statistical stationary states. Another type that is often found in applications is the self-similar behavior. In particular, there has been quite some interest in recent years on the scaling behavior of Hamilton-Jacobi-like growth models [64] and Cahn-Hilliard-like models for phase ordering and phase separation [14]. The rigorous treatment of such scaling results often hinges on well-posedness of the governing differential equation with rough initial data. Such a theory is available for the Hamilton-Jacobi equation, thanks to the work of Crandall and Lions [75]. As a result, most of the results on the scaling behavior for Hamilton-Jacobi-like growth models can be rigorously proved [85]. The scaling exponents often depends on the statistics of the maximum value of a large number of identically distributed random variables, which has been classified in the literature of probability theory [73]. For phase ordering and phase separation type of problems, on the other hand, the mathematical difficulties involved in defining solutions for the relevant governing equations (here the various forms of curvature flows) with rough initial data seem rather severe and rigorous treatment of physical theories such as the Lifshitz-Slyozov theory [14] does not seem to be in sight.

In this review, we have concentrated on dissipative PDEs and hence omitted work on invariant measures for Hamiltonian PDEs such as the nonlinear Schrödinger equation. We refer to $[\mathbf{1 3}, \mathbf{8 4}]$ for details on these results. Also omitted is the work on stochastic quantization, which is very relevant to our discussions on stochastic Ginzburg-Landau equation [26, 57].

We end this review with a list of open problems on the topics that we discussed.

1. On stochastic Burgers and Hamilton-Jacobi equations, the most pressing open problems seem to be the existence or nonexistence of an invariant measure on $\mathbb{R}^{n}$, and the hyperbolicity of the TSM in high dimensions. 
2. On the stochastic passive scalar equation, it is of great interest to describe geometrically the structure of the stationary passive scalar field. In particular, we are interested in characterizing the numerically observed "cliffs" that resulted in the saturation of the scaling exponents of the structure functions.

3. On the stochastic Navier-Stokes equations, from a technical point of view, it is highly desirable to fully develop the machinery of Malliavin calculus for PDEs. In connection with two dimensional turbulence, we would like to study the inviscid limit of the stationary solutions in the presence of large scale forcing and damping.

Also of interest is the problem of weak turbulence or wave turbulence. This concerns a large class of nonlinear dispersive wave equations such as the nonlinear Schrodinger equation. Even though the predictions are quite precise at a physical level, from a mathematical point of view, the problem is not very well formulated. It would be interesting even to define the rather framework under which the problem of weak turbulence can be studied in precise mathematical terms.

Acknowledgement: It is a pleasure to acknowledge the contributions from many people I have worked with, including K. Khanin, D. Liu, J. Mattingly, A. Mazel, Ya. Sinai and E. Vanden-Eijnden. My work is partially supported by a Presidential Faculty Fellowship from NSF.

\section{References}

[1] Batchelor, G.K., Computation of the energy spectrum in homogeneous two-dimensional turbulence, Phys. Fluids. Suppl. II, 12 233-239 (1969).

[2] Balkovsky, E., Lebedev, V., Instanton for the Kraichnan passive scalar problem, Phys. Rev. E, 58, 5776-5795 (1998).

[3] Barenblatt, G.I., Chorin, A.J., Scaling laws and vanishing viscosity limits for wall-bounded shear flows and for local structures in developed turbulence, Comm. Pure Appl. Math., 50, 381-398 (1997).

[4] Barenblatt, G.I., Chorin, A.J., Turbulence: an old challenge and new perspective, Meccanica, 33, 445-468 (1998).

[5] Barenblatt, G.I., Chorin, A.J., New Perspectives in Turbulence: Scaling Laws, Asymptotics, and Intermittency, SIAM Rev., 40, 265-291 (1998).

[6] Bec, J., Universality of velocity gradients in forced Burgers turbulence, preprint, 2001.

[7] Bec, J., Frisch, U., Khanin, K., Kicked Burgers turbulence, J. Fluid Mech., 416 239-267 (2000).

[8] Bernard, D., Gawȩdzki, K., and Kupiainen, A., Anomalous scaling in the N-point function sof a passive scalar, Phys. Rev. E, 54, 2564-2572 (1996).

[9] Billingsley, P., Convergence of Probability Measures, John Wiley \& Sons, Inc., New York (1968).

[10] Blatter, G., Feigelman, M. V., Geshkenbein, V. B., Larkin, A. I., and Vinokur, V. M., Vortices in high-temperature superconductors, Rev. Modern Phys., 66, 1125-1388 (1994).

[11] Boldyrev, S.A., Velocity-difference probability density functions for Burgers turbulence, Phys. Rev. E, 55, 6907-6910 (1997).

[12] Bouchaud, J.-P., and Mézard, M., Velocity fluctuations in forced Burgers turbulence, Phys. Rev. E, 54, 5116-5121 (1996).

[13] Bourgain, J., Nonlinear Schrödinger equations, in Hyperbolic Equations and Frequency Interactions, IAS/Park City Mathematics Series, 5, Caffarelli and E ed., American Mathematical Society (1999).

[14] Bray, A.J., Theory of phase-ordering kinetics, Advances in Physics, 43(3), 357-459 (1994).

[15] Brenier, Y., The Least Action Principle and the Related Concept of Generalized Flows for Incompressible Perfect Fluids, J. Amer. Math. Soc., 2, 225-255 (1989).

[16] Brenier, Y., The dual least action problem for an ideal, incompressible fluid, Arch. Rational Mech. Anal. 122, 323-351 (1993). 
[17] Bricmont, J., Kupiainen, A., and Lefevere, R., Exponential mixing of the 2D stochastic Navier-Stokes dynamics, preprint.

[18] Chertkov, M., Falkovich, G., Kolokolov, I., Lebedev, V., Statistics of a passive scalar advected by a large-scale two-dimensional velocity field: Analytic solution, Phys. Rev. E, 51, 56095627 (1995).

[19] Chertkov, M., Falkovich, G., Kolokolov, I., Lebedev, V., Normal and anomalous scaling of the fourth-order correlation function of a randomly advected passive scalar, Phys. Rev. E, 52(5), 4924-4941 (1995).

[20] Chorin, A. J., Vorticity and Turbulence, Springer, New York, 1994.

[21] Constantin, P., and Foias, C., Navier-Stokes Equations, University of Chicago Press, Chicago (1988).

[22] Constantin, P., E, W., and Titi, E., Onsager's conjecture on the energy conservation for solutions of Euler's equation, Comm. Math. Phys., 165, 207-209 (1994).

[23] Dafermos, C., Generalized characteristics and the structure of solutions of hyperbolic conservation laws, Indiana Univ. Math. J., 26, 1097-1119 (1977).

[24] DiPerna, R. and Majda, A.J., Oscillations and concentrations in weak solutions of the incompressible fluid equations, Comm. Math. Phys., 108, 667-689 (1986).

[25] DiPerna, R. and Lions, P.L., Ordinary differential equations, transport theory and Sobolev spaces, Invent. Math. 98, 511-547 (1989).

[26] Doering, C., Thesis, University of Texas, Austin.

[27] E, W., Aubry-Mather theory and periodic solutions of forced Burgers equations, Comm. Pure Appl. Math., LII 0811-0828 (1999).

[28] E, W., A class of homogenization problems in the calculus of variations, Comm. Pure Appl. Math, 44(7), 733-759 (1991).

[29] E, W., Khanin, K., Mazel, A., and Sinai, Ya. G., Probability distributions functions for the random forced Burgers equation, Phys. Rev. Lett., 78, 1904-1907 (1997).

[30] E, W., Khanin, K., Mazel, A., and Sinai, Ya. G., Invariant measures for the random-forced Burgers equation, Ann. Math., 151, 877-960 (2000).

[31] E, W. and Liu, D., Gibbsian dynamics and invariant measure for stochastic dissipative PDEs, submitted to J. Stat. Phys.

[32] E, W. and Mattingly, J., Ergodicity for the Navier-Stokes Equation with Degenerate Random Forcing: Finite Dimensional Approximation, to appear in Comm. Pure. Appl. Math.

[33] E, W., Mattingly, J. and Sinai, Ya., Gibbsian dynamics and ergodicity for the stochastically forced Navier-Stokes Equation, to appear in Comm. Math. Phys.

[34] E, W., and Vanden-Eijnden, E., Asymptotic theory for the probability density functions in Burgers turbulence, Phys. Rev. Lett., 83, 2572-2575 (1999).

[35] E, W., and Vanden-Eijnden, E., Statistical theory of the stochastic Burgers equation in the inviscid limit, Comm. Pure Appl. Math., LIII, 0852-0901 (2000).

[36] E, W., and Vanden-Eijnden, E., Generalized flows, intrinsic stochasticity and turbulent transport, Proc. Nat. Acad. Sci., 97(15), 8200-8205 (2000).

[37] E, W., and Vanden-Eijnden, E., Prandtl number effect in passive scalar advection, Physica $\mathrm{D}(2000)$.

[38] E, W., and Vanden-Eijnden, E., Generalized flows and transport equations, preprint.

[39] Eckmann, J. P., and Hairer, M., Uniqueness of the invariant measure for a stochastic PDE driven by degenerate noise, preprint.

[40] Evans, L.C., The perturbed test function method for viscosity solutions of nonlinear PDE, Proc. Roy. Soc. Edinburgh, Ser A III(3-4), 359-375 (1989).

[41] Eyink, G., Dissipation in Turbulent Solutions of $2 D$ Euler equations, Nonlinearity, 14, 787802 (2001).

[42] Fathi, A., Théorème KAM faible et théorie de Mather sur les systémes lagrangivens, C.R. Acad. Sci. Paris Sér. I Math. 324(9), 1043-1046 (1997).

[43] Feigelman, M. V., One-dimensional periodic structures in a weak random potential, Sov. Phys. JETP, 52, 555-561 (1980).

[44] Ferrario, B., Ergodic results for stochastic Navier-Stokes equation, Stochastics and Stochastics Reports, 60(3-4), 271-288 (1997).

[45] Flandoli, F., Dissipativity and invariant measures for stochastic Navier-Stokes equations, NoDEA, 1, 403-426 (1994). 
[46] Flandoli, F., Maslowski, B., Ergodicity of the 2-D Navier-Stokes equation under random perturbations, Comm. in Math. Phys., 171, 119-141 (1995).

[47] Foias, C., Manley, O., Rosa, R., Temam, R., Navier-Stokes Equations and Turbulence, to be published.

[48] Frisch, U., Turbulence: The Legacy of A. N. Kolmogorov, Cambridge University Press (1995).

[49] Frisch, U., Mazzino, A., Noullez, A., and Vergassola, M., Lagrangian method for multiple correlations in the passive scalar advection, cond-mat/9810074, submitted to Phys. Fluids (1998).

[50] Gawȩdzki, K., Kupiainen, A., Anomalous scaling of a passive scalar, Phys. Rev. Lett., 75, 3834-3837 (1995).

[51] Gawędzki, K., Vergassola, M., Phase transition in the passive scalar advection Physica D, 138, 63-90 (2000).

[52] Gotoh, T. and Kraichnan, R.H., Steady-state Burgers turbulence with large-scale forcing, Phys. Fluids 10, 2859-2866 (1998).

[53] Harris, T.E., The existence of stationary measures for certain Markov processes, in Proceedings of the Third Berkeley Symposium on Mathematical Statistics and Probability, 19541955, II, 113-124, University of California Press (1956).

[54] Ichihara, K. and Kunita, H., A classification of the second order degenerate elliptic operators and its probabilistic characterization, Z. Wahrscheinlichkeitstheorie und Verw. Gebiete, 30, 235-254 (1974).

[55] Iturriaga, R. and Khanin, K., Burgers turbulence and random Lagrangian systems, preprint.

[56] Jauslin, H. R., Kreiss, H. O., and Moser, J., On the forced Burgers equation with periodic boundary conditions, preprint (1997).

[57] Jona Lasinio, G. and Mitter, S.K., On the stochastic quantization of field theory, Comm. Math. Phys., 101, 409-436 (1985).

[58] Kardar, M., Parisi, G., Zhang, Y.C., Dynamic scaling of growing interfaces, Phys. Rev. Lett., 56, 889-892 (1986).

[59] Kolmogorov, A.N., The local structure of turbulence in incompressible viscous fluid for very large Reynolds numbers, C.R. (Doklady) Acad. Sci. URSS (N.S.), 30, 301-305 (1941a).

[60] Kolmogorov, A.N., On degeneration of isotropic turbulence in an incompressible viscous liquid, C.R. (Doklady) Acad. Sci. URSS (N.S.), 31, 538-540 (1941b).

[61] Kolmogorov, A.N., A refinement of previous hypothese concerning the local structure of turbulence in a viscous incompressible fluid at high Reynolds number, J. Fluid Mech., 13, 82-85 (1962).

[62] Kraichnan, R.H., Inertial ranges in two-dimensional turbulence, Phys. Fluids, 10, 1417-1423 (1967).

[63] Kraichnan, R.H., Dispersion of particle pairs in homogeneous turbulence, Phys. Fluids 9, 1937-1943 (1966).

[64] Krug, J. and Spohn, H., Kinetic roughening of growing surfaces, in Solids far from equilibrium (Godrèche, G.C., ed.), 477-582, Cambridge University Press, England (1992).

[65] Kuksin, S., Shirikyan, A., Stochastic Dissipative PDEs and Gibbs Measures, Comm. Math. Phys., 213, 291-330 (2000).

[66] Kuksin, S.B. and Shirikyan, A., On Dissipative Systems Perturbed by Bounded Random Kick-forces, preprint (2000).

[67] Kunita, H., Supports of diffusion processes and controllability problems, in Proceedings of the International Symposium on Stochastic Differential Equations (Res. Inst. Math. Sci., Kyoto Univ., Kyoto, 1976), 163-185, New York, Wiley, (1978).

[68] Kunita, H., Stochastic flows and stochastic differential equations Cambridge University Press, Cambridge (1990).

[69] Kunita, H., Stochastic differential equations, Cambridge University Press, Cambridge (1990).

[70] Kusuoka, S. and Stroock, D., Applications of the Malliavin calculus. II, J. Fac. Sci. Univ. Tokyo Sect. IA Math., 32(1), 1-76, (1985).

[71] Lax, P.D., Hyperbolic Systems of Conservation Laws and the Mathematical Theory of Shock Waves, SIAM, Philadelphia (1973).

[72] Le Jan, Y., Raimond, O., Integration of Brownian vector fields, preprint.

[73] Leadbetter, M., Lindgren, G. and Rootzen, H., Extremes and Related Properties of Random Sequences and Processes, Springer-Verlag, 1983.

[74] Lifshitz, I.M. and Slyozov, V.V., J. Phys. Chem. Solids, 19, 35 (1961). 
[75] Lions, P.L., Generalized Solutions of Hamilton-Jacobi Equations, Pitman Advanced Publishing Program (1982).

[76] Lions, P.L., Papanicolaou, G., Varadhan, S.R.S., Homogenization of Hamilton-Jacobi equations, unpublished.

[77] Majda, A.J., J. Stat. Phys., 73, 515-542 (1993).

[78] Majda, A.J., Kramer, P.R., Simplified models for turbulent diffusion: Theory, numerical modeling and physical phenomena, Phys. Rep., 314, 237-574 (1999).

[79] Masmoudi, N., and Young, L.-S., Ergodicity Theory of Infinite Dimensional Systems with Application to Dissipative Parabolic PDEs, preprint.

[80] Mather, J.N., Existence of quasi-periodic orbits for twist homeomorphisms of the annulus, Topology, 21, 457-467 (1982).

[81] Mattingly, J.C., The stochastically forced Navier-Stokes equations:energy estimates and phase space contraction, Ph.D. thesis, Princeton University (1998).

[82] Mattingly, J.C., Ergodicity of $2 D$ Navier-Stokes equations with random forcing and large viscosity, Comm. Math. Phys. 206(2), 273-288 (1999).

[83] Mattingly, J.C., Exponential convergence for the stochastically forced Navier-Stokes equations and other partially dissipative dynamics, preprint.

[84] McKean, H. and Vaninski, K., Statistical mechanics of nonlinear wave equations and Brownian motion with restoring drift: The petit and microcanonical ensembles, Comm. Math. Phys., 160, 615-630 (1994).

[85] Molchanov, S.A., Surgailis, D. and Woyczynski, W.A., The large-scale structures in the universe and quasi-Voronoi tessellation of shock fronts in forced inviscid Burgers turbulence in $\mathbb{R}^{d}$, Ann. Appl. Prob., 7, 200-228 (1997).

[86] Monin, A.S. and Yaglom, A.M., Statistical Fluid Mechanics: Mechanics of Turbulence, MIT Press, Cambridge (1975).

[87] Norris, J., Simplified Malliavin calculus, in Séminaire de Probabilités, XX, 1984/85, 101-130. Springer, Berlin, (1986).

[88] Onsager, L., Statistical hydrodynamics, Nuovo Cimento (Supplemento), 6, 279 (1949).

[89] Parisi, G., Frisch, U., Turbulence and Predictability in Geophysical Fluid Dynamics (Varenna, 1983) 83-87 (North-Holland, Amsterdam) (1985).

[90] Polyakov, A.M., Turbulence without pressure, Phys. Rev. E, 52, 6183-6188 (1995).

[91] Shnirelman, A.I., Geom. Funct. Anal., 4, 586-620 (1994).

[92] Shraiman, B., Siggia, E., Phys. Rev. E, 49, 2912-2927 (1994).

[93] Sinai, Ya., Two results concerning asymptotic behavior of solutions of the Burgers equation with force, J. Stat. Phys., 64, 1-12 (1992).

[94] Sinai, Ya., Burgers system driven by a periodic stochastic flow, in Itô's Stochastic Calculus and Probability Theory, Springer-Verlag, New York, Berlin 347-355 (1996).

[95] Smoller, J., Shock Waves and Reaction Diffusion Equations, Springer-Verlag, New York, Berlin (1983).

[96] Stroock, D. and Varadhan, S.R.S., Multidimensional Diffusion Processes, Springer-Verlag, Berlin, New York (1979).

[97] Tadmor, E., $L^{1}$-stability and error estimates for approximate Hamilton-Jacobi solutions, UCLA CAM Report 98-21.

[98] Tartar, L., Systems of nonlinear partial differential equations, NATO Adv. Sci. Inst. Ser. C: Math. Phys. Sci., (Oxford, 1982) 263-285, 111 (Reidel, Dordrecht-Boston) (1983).

[99] Woyczynski, W.A., Burgers-KPZ Turbulence, Lecture Notes in Mathematics, 1700, SpringerVerlag (1998).

[100] Vishik, M. and Fursikov, A., Mathematical problems of statistical hydrodynamics, Kluwer Academic Publishers, (1988).

[101] Vol'pert, A., The space BV and quasilinear equations, Math. USSR, Sb., 2 225-267 (1967).

Department of Mathematics and, Program in Applied and Computational Mathematics, Princeton University, Princeton NJ 08544 
